\title{
Three-Dimensional Storm Structure and Low-Level Boundaries at Different Stages of Cyclic Mesocyclone Evolution in a High-Precipitation Tornadic Supercell
}

\author{
Daniel P. Betten, ${ }^{1}$ Michael I. Biggerstaff $\mathbb{D D}^{1,2,3}$ and Conrad L. Ziegler ${ }^{4}$ \\ ${ }^{1}$ School of Meteorology, University of Oklahoma, Norman, OK 73072, USA \\ ${ }^{2}$ Cooperative Institute for Mesoscale Meteorological Studies, University of Oklahoma, Norman, OK 73072, USA \\ ${ }^{3}$ Advanced Radar Research Center, University of Oklahoma, Norman, OK 73072, USA \\ ${ }^{4}$ National Severe Storms Laboratory, National Oceanic and Atmospheric Administration, National Weather Center, \\ Norman, OK 73072, USA \\ Correspondence should be addressed to Michael I. Biggerstaff; drdoppler@ou.edu
}

Received 31 August 2017; Revised 10 December 2017; Accepted 20 December 2017; Published 13 February 2018

Academic Editor: Stefano Federico

Copyright (C) 2018 Daniel P. Betten et al. This is an open access article distributed under the Creative Commons Attribution License, which permits unrestricted use, distribution, and reproduction in any medium, provided the original work is properly cited.

\begin{abstract}
Nearly continuous wind retrievals every three minutes for an unprecedented 90-minute period were constructed during multiple mesocyclone cycles in a tornadic high-precipitation supercell. Asymptotic contraction rate analysis revealed the relationship between the primary and secondary rear-flank gust fronts (RFGF and SRFGFs) and the rear-flank downdraft (RFD) and occlusion downdrafts. This is thought to be the first radar-based analysis where the relationship between the near-surface gust fronts and their parent downdrafts has been explored for sequential mesocyclones. Changes in the SRFGFs were associated with surges in the RFD. During part of the mesocyclone lifecycle, the SRFGF produced a band of low-level convergence and associated deep updraft along the southwestern side of the hook echo region that ingested the RFD outflow and limited both entrainment into the RFD and reinforcement of low-level convergence along the leading edge of the primary RFGF. The second mesocyclone intensified from stretching in an occlusion updraft rather than in the primary updraft. This low-level mesocyclone remained well separated from the updraft shear region vorticity that was associated with a more traditional midlevel mesocyclone. However, the third mesocyclone initiated in the vorticity-rich region of the primary updraft zone and was amplified by stretching in the primary updraft.
\end{abstract}

\section{Introduction}

Despite observations that suggest there might be few kinematic differences between tornadic and nontornadic lowlevel mesocyclones [1, 2], more than half of low-level mesocyclones do not produce tornadoes [3]. Additionally, while some storms only produce a single mesocyclone, other supercell storms produce several mesocyclones (i.e., "cyclic supercells," [4]), during which the cycle duration and intensity can vary greatly [5-11]. Some cyclic storms have been observed to only produce tornadoes after many cycles $[7,8]$, either as a natural progression of storm-scale airflow changes between cycles or as a result of the surrounding environment becoming more favorable for tornadogenesis.
Nonetheless, the occurrence of cyclic tornadic storms, those that produce tornadoes during almost every mesocyclone cycle $[4,6,12-16]$, implies the continual presence of conditions favorable for repeated tornadogenesis. Therefore, it is important to find ubiquitous storm-scale flow structures associated with cyclically tornadic supercells that may be absent in nontornadic cyclic supercells or noncyclic tornadic supercells $[7,8]$.

Based on early radar analyses and visual storm observations, Lemon and Doswell III [17] identified key stormscale structures in tornadic supercell storms: a mesocyclone embedded in both a deep updraft and a rear-flank downdraft (RFD), and an additional persistent downdraft in the forward flank region (FFD). Gust fronts were also depicted at the 
leading edge of the downdraft outflows with the rear-flank gust front (RFGF) propagating out beneath the mesocyclone as the RFD expanded. As more radar-based analyses and thermodynamic observations were collected, their conceptual model was later modified. Observational $[5-7,18]$ and numerical studies $[9,11]$ extended the conceptual model to account for cyclic mesocyclogenesis and the persistence of the primary updraft rather than cyclic redevelopment of the primary updraft as described by Lemon and Doswell III [17].

Perhaps the greatest recent modification made to the Lemon and Doswell III [17] conceptual model was to the storm-scale structure of near-surface gust fronts. Instead of a persistent, forward flank gust front (FFGF), Dowell and Bluestein [6] and Beck et al. [7] noted weak convergence boundaries extending north from the circulation [6] or only present above the surface [7]. While these radar analyses span multiple mesocyclone cycles, it has been much more difficult to gather surface observations from multiple mesocyclone cycles. Nevertheless, surface observations from individual cycles suggest that tornadic supercells tended to have weaker cold pools regardless of whether they had strong or nonexistent forward flank convergence boundaries [6, 19-22].

Another detail that has been added to the conceptual model has been the presence of a smaller-scale dynamically driven "occlusion" downdraft described as a separate downdraft from the primary RFD [23]. The occlusion downdraft is a ubiquitous flow feature found in several radar-based analyses with sufficient resolution to resolve the downdraft, which may only be a few kilometers wide $[2,6,21,24-$ 29]. Internal downdraft outflows have been observed at the surface in close proximity to tornadoes with highly variable thermodynamic structure [30-36]. These regions have also been observed in radar-based analyses being manifested as a secondary, rear-flank gust front (SRFGF, $[29,37-40]$ ) with rapidly evolving flow [41]. Radar-based ensemble Kalman Filter (EnKF) analyses [42] have suggested that the internal surges were associated with a dynamically induced downdraft, similar to but distinct from the traditional occlusion downdraft. Conversely, numerical simulations have found the internal surges to be the result of downdrafts driven by precipitation-loading [43] or midlevel flow stagnation [44]. It is unclear whether the SRFGF divides two portions of the occlusion downdraft or if it is associated with a separate secondary RFD [29], a distinct downdraft region not associated with the primary RFD, or the occlusion downdraft.

This study will present radar analyses over a 90-minute period, covering parts of three mesocyclone cycles of the 29 May 2004 Geary, OK, supercell, a prolific tornadoproducing storm. Here, we focus on documenting the internal storm structure and low-level boundaries at different stages of mesocyclone evolution to aid in developing contrasts between observed cyclic tornadic supercells and nontornadic supercells. The term "boundaries" herein refers to a distinct zone in which the character of the storm-relative flow changes. The boundaries may be kinematic, like the RFGF where the flow normal to the boundary ceases, or may be due to more subtle persistent deformation that altered the observed flow behavior. Additionally, for the first time, the relationship between the SRFGF, RFD, and occlusion downdraft was examined for three sequential mesocyclones. It was found that the SRFGF was mainly driven by RFD pulses but was augmented by occlusion downdrafts near the mesocyclone on the southern and eastern side of the circulation during the occlusion stage. At low levels, the SRFGF and primary RFGFs remained separated as the outflow from the SRFGF contributed to a strong region of convergence that led to an updraft band behind the primary RFGF during the mature stage of the second mesocyclone cycle. Hence, the outflow associated with the SRFGF was forced upward and reingested into the updraft before it could be advected towards the leading edge of the primary RFGF.

\section{Data Collection and Methodology}

On the afternoon of 29 May 2004, during the TELEX field experiment [45], two Shared Mobile Atmospheric Research and Teaching (SMART, [46]) radars observed a tornadic, high-precipitation supercell near Geary, OK, for about three hours covering the dissipating stage of one mesocyclone, the entire lifecycle of a second mesocyclone, and the organizing stage of a third mesocyclone (Figure 1). Herein the stages of mesocyclone evolution follows Burgess et al. [5], with the distinction that we refer to the period when the outflow from the RFD begins to push the primary RFGF away from the circulation as the "occlusion stage" rather than the dissipating stage.

Many aspects of the deployment were optimal for retrieving and examining the storm-scale kinematic structure, including a slow storm motion $\left(12 \mathrm{~m} \mathrm{~s}^{-1}\right)$, a large storm (60 km long), a long mesocyclone cycle frequency (90 minutes), and a large baseline $(40 \mathrm{~km})$ that allowed the storm to stay within the region in which dual-Doppler wind retrievals [47-49] could be constructed for a long period of time. The radars operated in sector-scan mode, collecting $120^{\circ}$ azimuthal swaths of data from 0.5 to $59^{\circ}$ in elevation over a $\sim 2.5$ minute period with a repeat cycle of three minutes. Nine of these volume scans, ranging in time from 2320 to 0052 UTC, were analyzed in the cloud electrification study by Calhoun et al. [50]. Here, 23 volume scans from 2358 to 0120 UTC have been analyzed. Their temporal coverage is illustrated in Figure 1.

The radar data were interpolated onto a Cartesian grid $(100 \mathrm{~km} \times 90 \mathrm{~km}$ with a $0.75 \mathrm{~km}$ horizontal spacing and 0.25 to $18.25 \mathrm{~km}$ AGL in the vertical with $0.5 \mathrm{~km}$ spacing) using a hybrid Cressman [51] and natural neighbor [52] weighting scheme. The wind synthesis was performed using the National Center for Atmospheric Research (NCAR) software package Custom Editing and Display of Reduced Information in Cartesian Space (CEDRIC; [53]) following a procedure similar to that of Palucki et al. [54]. To maintain consistent resolvable scales throughout the 90-minute period of the analysis, the wind retrievals employed a two-step Leise [55] filter which dampened wavelengths less than $4.5 \mathrm{~km}$ and eliminated wavelengths less than $3.0 \mathrm{~km}$. Changes in the storm structure are, thus, related to evolution and are not a reflection of the storm's varying range from the radars.

Additionally, an environmental sounding unit stayed ahead of the storm as it moved eastward into the horizontally 
SMART-radar volumetric coverage: $2350-0120$ UTC

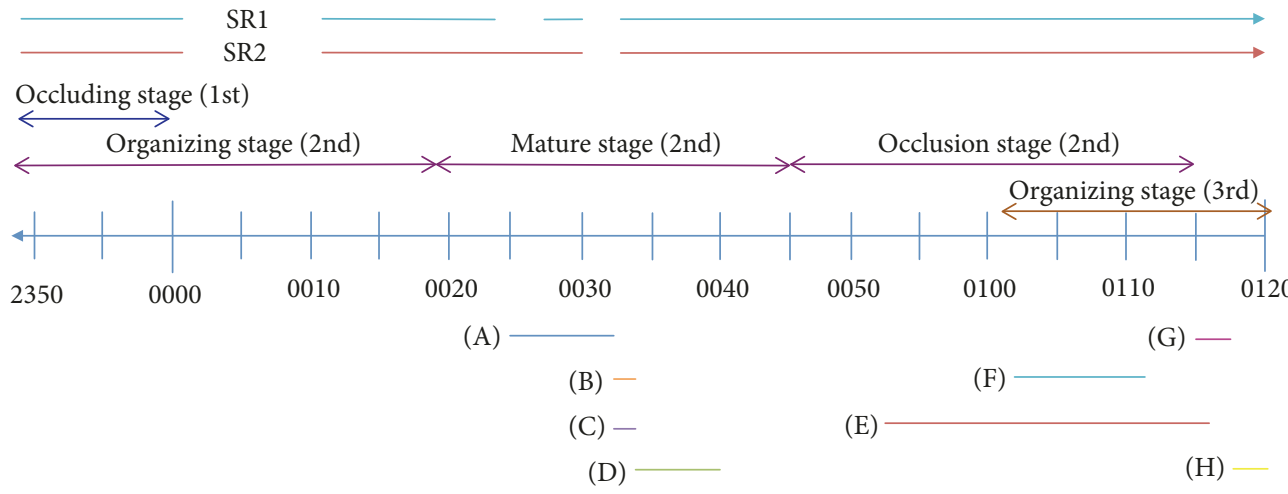

DOW observed tornadoes

FIGURE 1: Timeline for SMART-radar observations, mesocyclone lifecycles, and tornado lifecycles (marked from (A) to (H) as indicated by a DOW radar that was close to the hook echo).

heterogeneous stable, cool, and moist boundary layer in westcentral Oklahoma [45]. Environmental inflow soundings were obtained at 2236 UTC on 29 May and at 0008 and 0130 UTC on 30 May 2004.

Cohen and Schultz [56] demonstrated that baroclinic boundaries should be manifested where initially weak buoyancy gradients are magnified along persistent confluent zones. The instantaneous asymptotic contraction rate, a solely kinematic quantity, was shown to reveal where baroclinic boundaries developed as deformation and rotation were allowed to act on weak baroclinic gradients. According to [56], the asymptotic contraction rate is an estimate of the rate at which the horizontal distance between two adjacent parcels decreases. Mathematically, the asymptotic contraction rate, $C$, is given by

$$
C=\frac{1}{2}\left[-D+\left(E^{2}-\zeta^{2}\right)^{1 / 2}\right], \quad E \geq|\zeta|
$$

where $D$ is divergence and $E$ is the deformation determinant (defined by equation (2)) of the stretching deformation, $E_{\mathrm{st}}$, given by (3) and the shearing deformation, $E_{\mathrm{sh}}$, given by (4). The vertical vorticity is denoted as $\zeta$.

$$
\begin{aligned}
E & =\left(E_{\mathrm{st}}^{2}+E_{\mathrm{sh}}^{2}\right)^{1 / 2}, \\
E_{\mathrm{st}} & =\frac{\partial u}{\partial y}-\frac{\partial v}{\partial x} \\
E_{\mathrm{sh}} & =\frac{\partial u}{\partial y}+\frac{\partial v}{\partial x}
\end{aligned}
$$

According to (1), the contraction rate is only valid when deformation exceeds the magnitude of vorticity, allowing for an air parcel to align with an axis of zero rotation. To find boundaries in the Geary, OK, supercell, the contraction rate was calculated at $250 \mathrm{~m}$ above ground level. The contraction rate is only a valid indicator of baroclinic boundaries if the enhanced regions are persistent long enough for the air parcels to converge and tighten the buoyancy gradient.
The boundary inferences also assume that buoyancy is not substantially changing along the air parcel path.

The applicability of the instantaneous asymptotic contraction rate was tested through a comparison with a thermodynamic retrieval (not shown) using a diabatic Lagrangian analysis (DLA, [57]) for the 9 June 2009 case from Ziegler [58]. Following the guidance of Cohen and Schultz [56], the contraction rate compared favorably with the low-level thermodynamic boundaries revealed by the DLA. A future study will examine the thermodynamic structure of this case in more depth using a DLA. In this study, the contraction rate, along with changes in flow behavior, is used to define the location of a boundary, even though the implied buoyancy gradient may be weak at that time. In this manner, the instantaneous influence of vertical drafts on low-level evolution can be readily diagnosed.

\section{Storm Overview}

On 29 May 2004 storms initiated along a dryline in far western Oklahoma at approximately 2130 UTC. Prior to obtaining low-level rotation, storm motion was towards the northeast. As the southernmost cell gained low-level rotation ( 2245 UTC), the mean storm motion shifted towards the east-northeast. This storm, hereafter referred to as the Geary storm, evolved into a high-precipitation supercell [59] and began cyclically producing tornadoes at 2333 UTC and continued to produce tornadoes until 0651 UTC. The storm produced a total of 18 tornadoes and numerous large hail reports, with several reports over 4 inches in diameter [60]. Additionally, according to observations by a mobile Doppler on Wheels (DOW; [61]), a total of eight tornadoes (or tornado-like vortices), including one that lasted 24 minutes and two anticyclonic tornadoes, were observed during the 90minute period of interest in this study (Figure 1). Calhoun et al. [50] provide additional discussion of the mesoscale environment and evolution of the Geary supercell.

The storm-scale environment during the observational period is represented by a Mobile GPS Advanced Upper-Air 


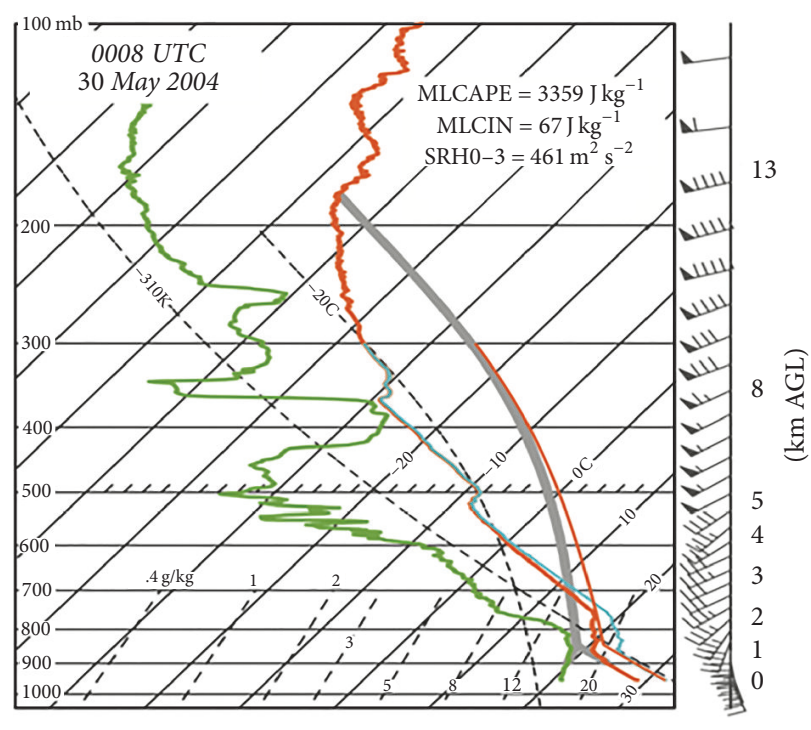

(a)

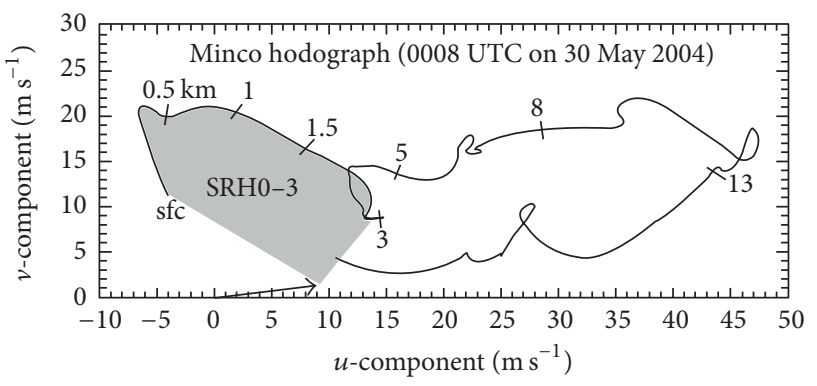

(b)

Figure 2: MGAUS sounding in Minco, OK, at 0008 UTC on 30 May 2004. In (a) is the thermodynamic sounding with parameters in the upper right-hand corner. In (b) is the hodograph with the $0-3 \mathrm{~km}$ storm-relative helicity shaded in grey and an arrow indicating the average storm motion.

Sounding (MGAUS) system sounding launched $75 \mathrm{~km}$ southeast of the storm near Minco, OK, at approximately 0008 UTC (Figure 2). The sounding contained large amounts of instability, with a mixed-layer convective available potential energy (MLCAPE) of $\sim 3300 \mathrm{~J} \mathrm{~kg}^{-1}$, while a substantial stable layer remained between $850 \mathrm{mb}$ and $750 \mathrm{mb}$ with a mixedlayer convective inhibition (MLCIN) of $67 \mathrm{~J} \mathrm{~kg}^{-1}$. It is possible that the strength of the stable layer, in addition to the strong low-level storm-relative winds, aided the longevity of the storm by preventing the outflow from surging ahead of the main updraft [62]. The $0-3 \mathrm{~km}$ storm-relative helicity (SRH0-3) was also extremely high at $461 \mathrm{~m}^{2} \mathrm{~s}^{-2}$, well above the threshold seen in strongly tornadic supercell environments [63]. More generally, the mobile storm-inflow soundings obtained during the period 2236-0130 UTC consistently found large values of MLCAPE and SRH0-3, in excess of $3200 \mathrm{~J} \mathrm{~kg}^{-1}$ and $400 \mathrm{~m}^{2} \mathrm{~s}^{-1}$, respectively. Interestingly, there was a significant weakness in the wind profile between 2 and $5 \mathrm{~km}$ where the zonal component was nearly constant and the meridional component weakened $6 \mathrm{~m} \mathrm{~s}^{-1}$.

\section{Kinematic Structure at Different Stages of the Mesocyclone Lifecycle}

\subsection{Organizing Stage of the Second Mesocyclone: 2358-0022 UTC}

4.1.1. Low-Level Boundaries. At 2358 UTC, the Geary supercell was undergoing cyclic mesocyclogenesis $[5-7,18]$. The old occluded circulation (Figure 3(a), $x=-63, y=28$ ) and the associated precipitation core (Figure $3(\mathrm{~b})$ ) were located northwest of the new, organizing mesocyclone (Figure 3(a), $x=-53, y=24)$ consistent with a stage 3 structure in the conceptual model of Beck et al. [7]. During the organizing stage, the primary rear-flank gust front (RFGF, solid red line in Figure 3(a)) was draped along the southern periphery of the storm outflow on the storm's southern flank. Meanwhile, secondary rear-flank gust fronts (SRFGFs) outlined the edge of the occlusion downdraft outflow associated with the occluding circulation (solid dark blue line) and the new rainy downdraft (dashed light blue line), which developed on the north side of the developing circulation.

As the occluding circulation retreated, in a storm-relative sense, to the northwest side of the storm, the associated SRFGF expanded southward, such that the southern end merged with the primary RFGF (Figure 3(a)). By 0011 UTC (Figure 3(c)), the new SRFGF had surged westward, overtaking the old outflow region, as the RFD strengthened (Figure 3(d), $x=-54, y=27$ ) and the low-level updraft zone and vertical vorticity field became better organized (Figures $3(\mathrm{~b})$ and $3(\mathrm{~d})$ ). Interestingly, the primary RFGF near the developing circulation did not surge southward until the end of the organizing stage (0022 UTC), waiting instead for an occlusion downdraft to develop and strengthen on the south side of the circulation (Figure 3(f)).

On the eastern edge of the rainy downdraft (Figure 3(f) $x=-42, y=26$ ), persistent positive asymptotic contraction was noted extending northeastward through the forward flank of the storm and will hereafter be referred to as the forward flank convergent boundary (FFCB, dashed green line in Figure 3). We have chosen to distinguish it from the left-flank convergent boundary found by Beck and Weiss [64] because it was located in the forward flank and appears too weak to be the delineating boundary between modified inflow and cold RFD outflow, as was found in 


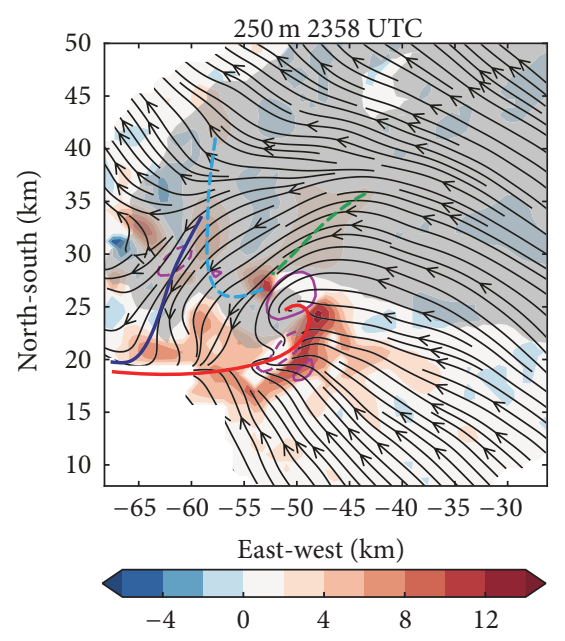

(a)

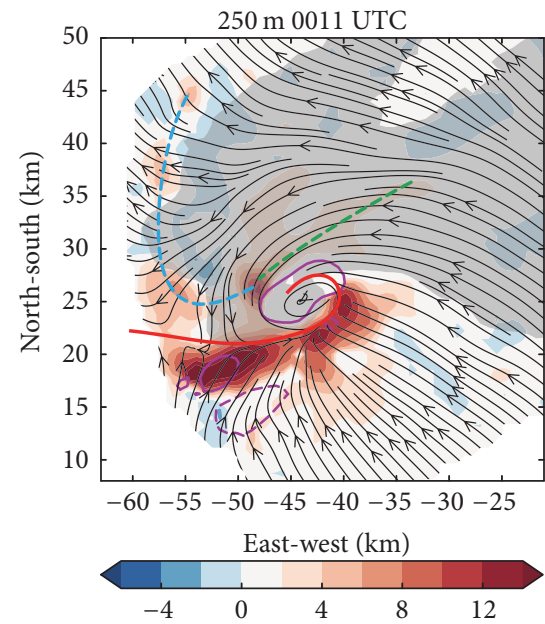

(c)

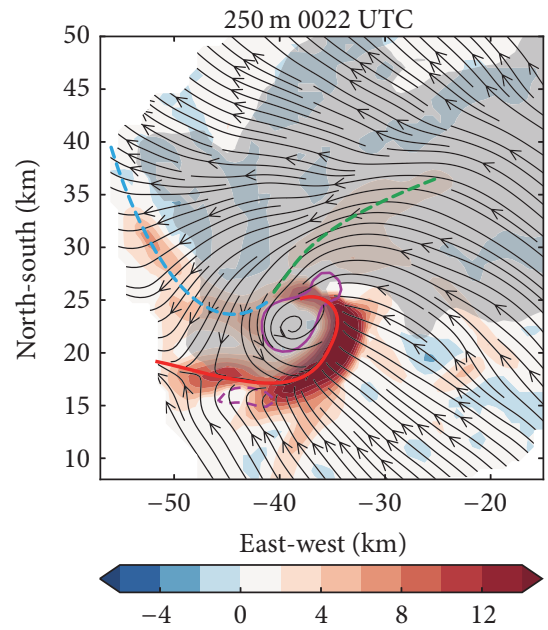

(e)

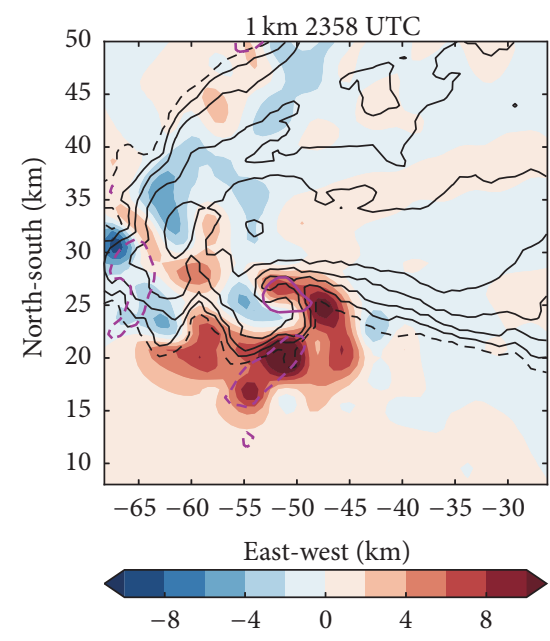

(b)

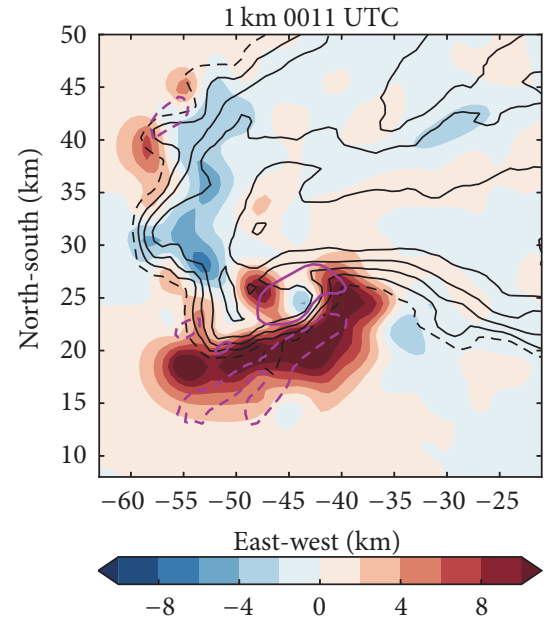

(d)

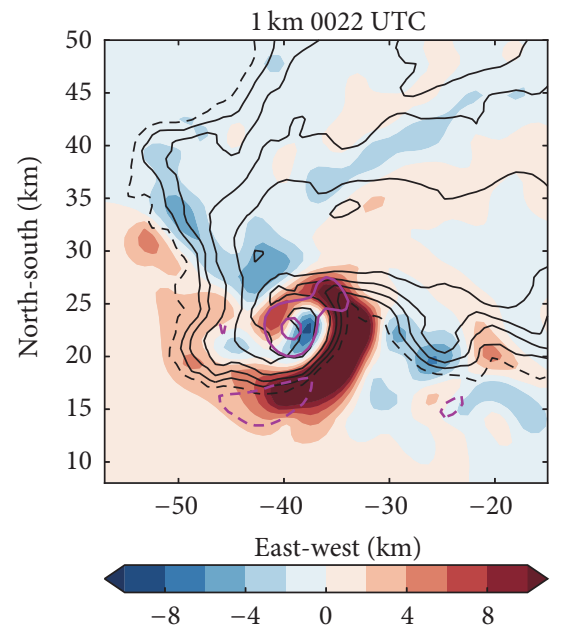

(f)

FIgURE 3: Storm-relative streamlines at an altitude of $250 \mathrm{~m} \mathrm{(a,} \mathrm{c,} \mathrm{e)} \mathrm{at} 2358$ UTC (a), 0011 UTC (c), and 0022 UTC (e) with the asymptotic contraction rate overlaid in color in units of $10^{-3} \mathrm{~s}^{-1}$ according to the color scale. Near-surface boundaries are drawn with the primary rearflank gust front (RFGF) in red, the secondary rear-flank gust front (SRFGF) in light blue, the previous mesocyclone cycle's secondary rearflank gust front in dark blue, and the forward flank convergence boundary (FFCB) in green. Reflectivity greater than $40 \mathrm{dBZ}$ is shaded in grey. Vertical vorticity maxima are annotated with magenta contours at -5 (dashed), 10 , and 30 (solid) $\times 10^{-3} \mathrm{~s}^{-1}$. Horizontal cross-sections at $1 \mathrm{~km}$ altitude (b, d, f) for the 2358 UTC (b), 0011 UTC (d), and 0022 UTC (f) analysis of vertical velocity (in $\mathrm{m} \mathrm{s}^{-1}$, according to the color scale), radar reflectivity greater than $20 \mathrm{dBZ}$ (contoured every $10 \mathrm{~dB}$ with $20 \mathrm{dBZ}$ dashed and higher values solid). Vertical vorticity maxima are annotated in magenta at -5 (dashed), 10 , and 30 (solid) $\left(\times 10^{-3} \mathrm{~s}^{-1}\right)$. 
their study. Moreover, despite the presence of a midlevel forward flank downdraft, a distinct forward flank gust front was never present in the analysis-similar to other supercell studies $[6,7,31,41]$. At this time, only portions of the FFCB demonstrated enough convergence to classify it as a boundary. Eventually, this boundary was collocated with enhanced convergence during the occlusion stage. Though oriented towards the northeast, the FFCB remained on the northwest side of the circulation as the vortex expanded to the southwest and increased in asymmetry (Figure 3(f)).

4.1.2. Vertical Draft Evolution. As the mesocyclone progressed through the organizing stage, the downdraft structure changed significantly. The downdrafts in the rear-flank region (Figure 3(b), $x=-63, y=24$; see also Figures $4(a)$ and 4(c)) were at their weakest point during mesocyclogenesis. Indeed, rising motion on the east side of the occluded circulation divided pockets of sinking motion associated with the old circulation and the new hook echo (Figure 3(b), $x=-55$, $y=24)$. By 0011 UTC, a centralized low-level RFD region had developed (Figure 3(d), $x=-53, y=27$ ). Initially, the RFD was shallow. By 0022 UTC, however, the RFD was part of a deep region of subsidence that extended up to middle levels (Figure 4(e)).

Between 0011 and 0022 UTC, the occlusion downdraft (Figure 3(d), $x=-45, y=25$, and Figure 3(f), $x=-39, y=$ 22) doubled in strength while remaining separated from the RFD by the occlusion updraft to its northwest. This separation can best be seen when the vertical motion field is viewed three-dimensionally (Figure 4(e)). Furthermore, the SRFGF (Figure 3(e)) was clearly positioned between the RFD and occlusion downdraft regions, suggesting that the potentially colder RFD air was not being advected into the low-level circulation at this time.

During the development of the second mesocyclone (2358 UTC), the updraft straddled the hook echo at low and middle levels (Figure 3(b)), coincident with weak outflow in the new hook echo region. The western part of the updraft zone appeared to be connected to the older occluding circulation, as suggested by the secondary inflow notch on the western edge of the primary hook echo. However, as the new RFD strengthened and the SRFGF surged southward (Figure 3(d)), the secondary inflow notch filled with precipitation. Convergence also became stronger and more continuous along the western end of the RFGF (Figure 3(c), $x=-58$ to $-45, y=18$ ). The enhanced convergence resulted in a strong, elongated low-level updraft. By 0022 UTC, the gust front bulged out towards the southeast, shifting the strongest convergence and low-level updraft eastward, coincident with the intensification of the low-level occlusion downdraft (Figure 3(f), $x=-35, y=20$ ).

4.1.3. Evolution of Vertical Vorticity. Initially at 2358 UTC, the developing low-level mesocyclone vortex (Figure 3(b), $x=-53, y=25)$ was small, symmetric, and collocated with an updraft while also decreasing in size with height (Figure 4(b)). Indeed, the low-level mesocyclone was initially separate from the midlevel mesocyclone but appeared to deepen during the organizing period (Figures 4(d) and $4(\mathrm{f})$; see also [27]). The deepening was associated with stretching of vorticity outside the primary updraft. At 0011 UTC, the western part of the low-level mesocyclone at $1 \mathrm{~km}$ (Figure 3(d), $x=-48, y=24$ ) was coincident with an updraft that was disconnected from the primary updraft region. Stretching in this western updraft resulted in the main mesocyclone vortex becoming a distinct circulation from the vorticity collocated with the primary updraft farther east (Figure 4(f)). This evolution contrasts the more common mode in which upward growth of the low-level circulation occurs through stretching in the primary updraft with connection to the traditional midlevel mesocyclone via vortex line surgery [cf. [65]]. While not evolving in a traditional manner, similar dual midlevel vorticity structures have been observed in previous radar-based analyses $[6,24,27,66]$.

Both regions of vorticity evolved separately in time and will be distinguished as the mesocyclone vortex, or just mesocyclone (Figure 3(f), $x=-38, y=23$ ), and the updraft shear region vorticity (Figure 3(f), $x=-33, y=23$ ). During the middle of the organizing stage, the mesocyclone was broadest at low levels (Figure 4(d)), decreased in size with height, and was tilted towards the west-southwest. However, following a RFD surge at 0019 UTC, the mesocyclone became much more consistent in size and strength with height (Figure 4(f)) and became centered on the vertical velocity gradient (Figure 3(f), $x=-40, y=22$ ) producing a divided vortex structure at 0022 UTC. This transition in mesocyclone behavior is consistent with the Lemon and Doswell III [17] conceptual model of mesocyclone evolution between organizing and mature stages.

\subsection{Mature Stage of the Second Mesocyclone: 0027-0039 UTC}

4.2.1. Low-Level Boundaries. As the storm progressed into the mature stage, the next RFD intensification at 0027 UTC (Figure 5(b), $x=-39, y=27$ ) caused the SRFGF to also surge southward (Figure 5(a), $x=-38, y=20$ ) along the western edge of the mesocyclone circulation. During this period, the SRFGF boundary became well defined by asymptotic contraction, with rates approaching $10^{-2} \mathrm{~s}^{-1}$. After the initial southward surge between 0022 and 0027 UTC, the SRFGF and associated zone of enhanced asymptotic contraction rate did not progress any further south over the next 12 minutes. Furthermore, there was a delay between the southward surge in the SRFGF and changes in the position of the primary RFGF. The delay implies that different downdraft flow regimes were reinforcing the primary and secondary RFGFs, potentially delaying the subsequent occlusion of the second mesocyclone. Following the RFD surge, the asymptotic contraction in the southern portion of the FFCB briefly intensified (Figure 5(c), $x=-32, y=27$ ) creating a kinematic boundary that was straddled by moderate updraft and downdraft (Figure 5(d)).

4.2.2. Vertical Draft Evolution. Part of the reason the cold pool behind the SRFGF did not contribute directly to the primary RFGF cold pool during the mature stage of the second mesocyclone was that the RFD surge at 0027 UTC led to the development of a northwest-southeast-oriented band 


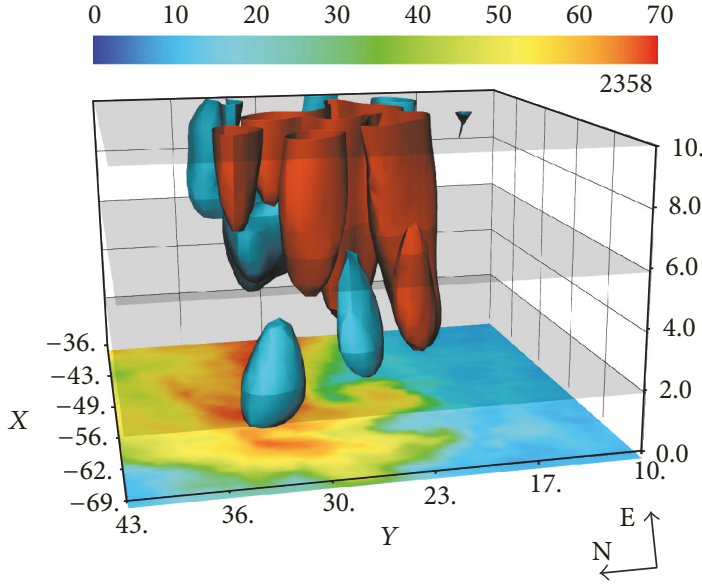

(a)

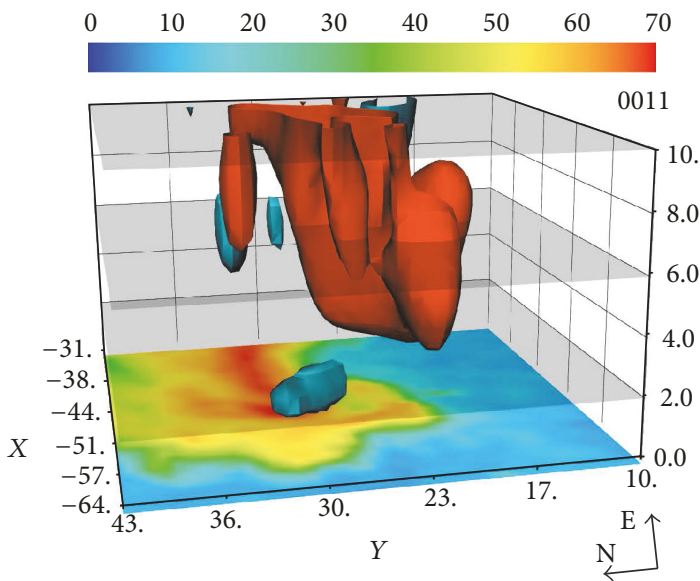

(c)

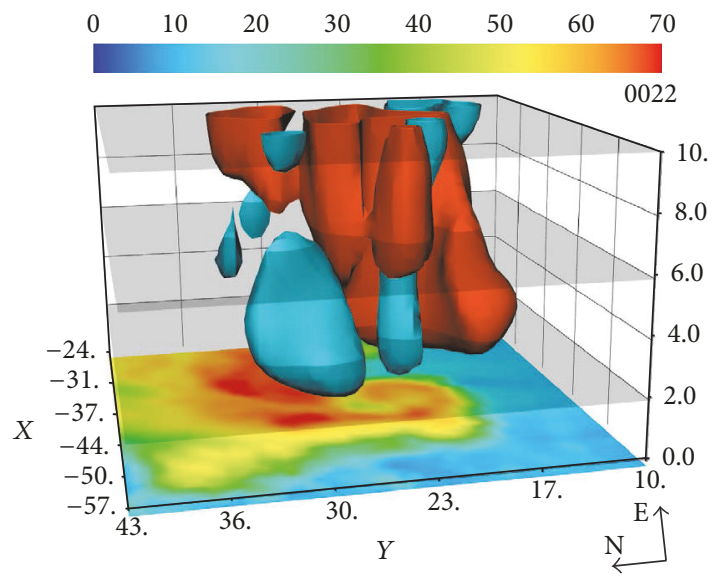

(e)

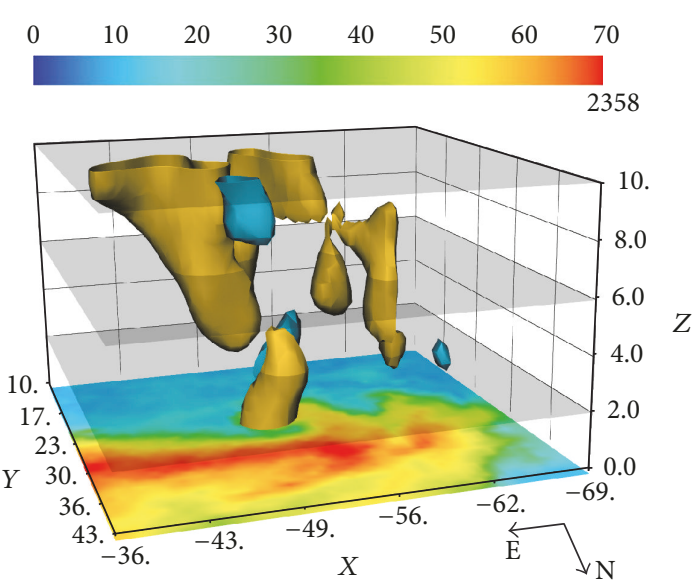

(b)

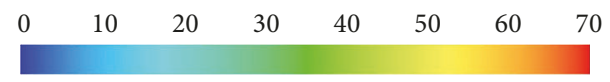

0011

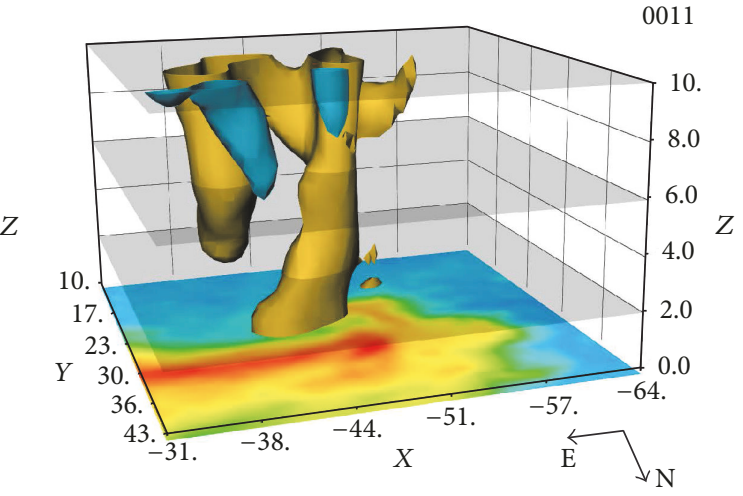

(d)

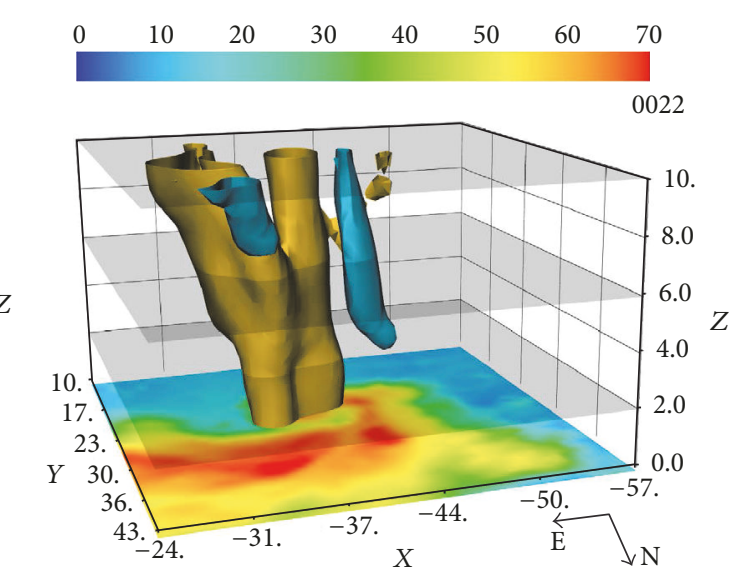

(f)

Figure 4: Three-dimensional isosurfaces of vertical velocity $\left(-5 \mathrm{~m} \mathrm{~s}^{-1}\right.$ [blue] and $20 \mathrm{~m} \mathrm{~s}^{-1}$ [red], (a), (c), (e)) and vertical vorticity $(-1 \times$ $10^{-3} \mathrm{~s}^{-1}$ [blue] and $1 \times 10^{-3} \mathrm{~s}^{-1}$ [gold], (b), (d), (f)) for 2358 UTC (a, b), 0011 UTC (c, d), and 0022 UTC (e, f). Radar reflectivity (in dBZ according to the color scale) at $1 \mathrm{~km}$ altitude is plotted at the bottom of each panel. Horizontal planes at 2,6 , and $10 \mathrm{~km}$ are shaded grey for reference. Note that panels (a), (c), and (e) are oriented with a perspective of looking at the storm from approximately the west while panels (b), (d), and (f) are oriented with a perspective of looking at the storm from approximately the north. 


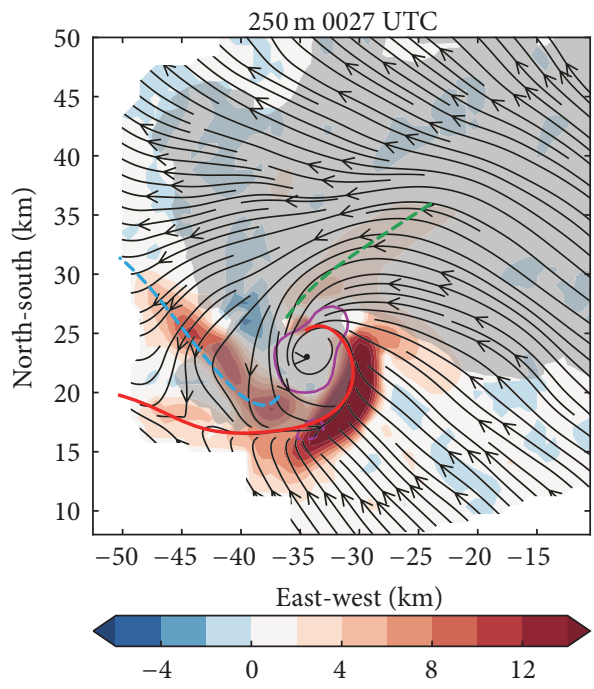

(a)

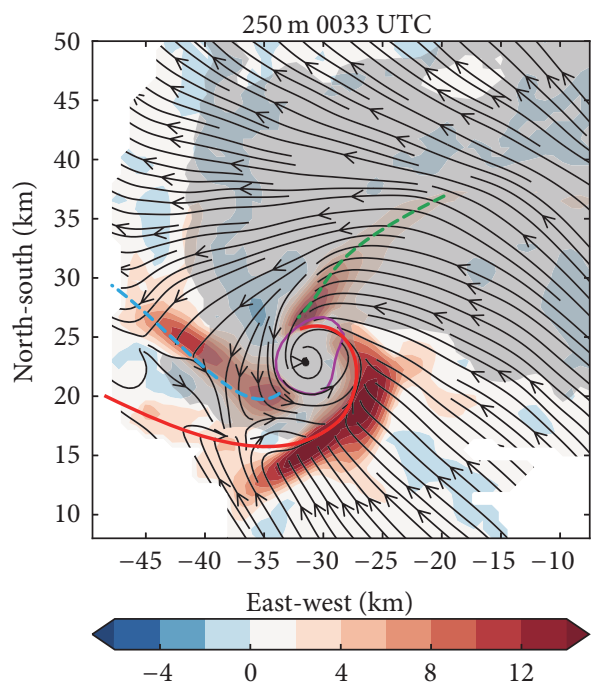

(c)

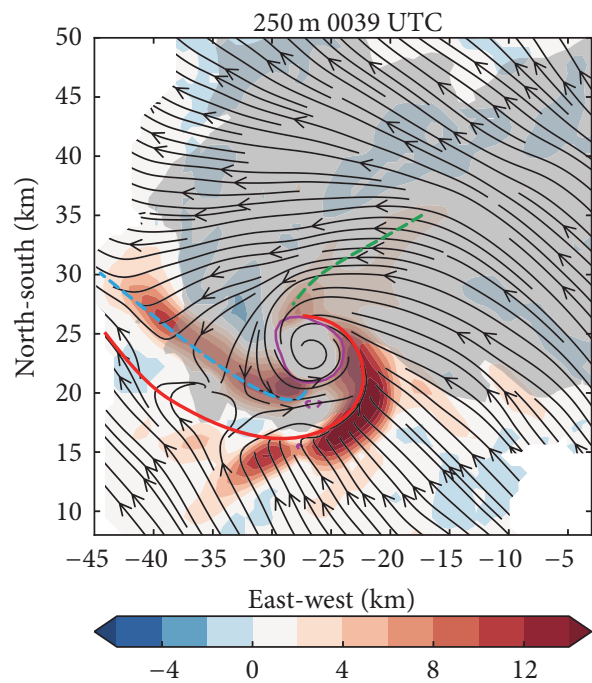

(e)

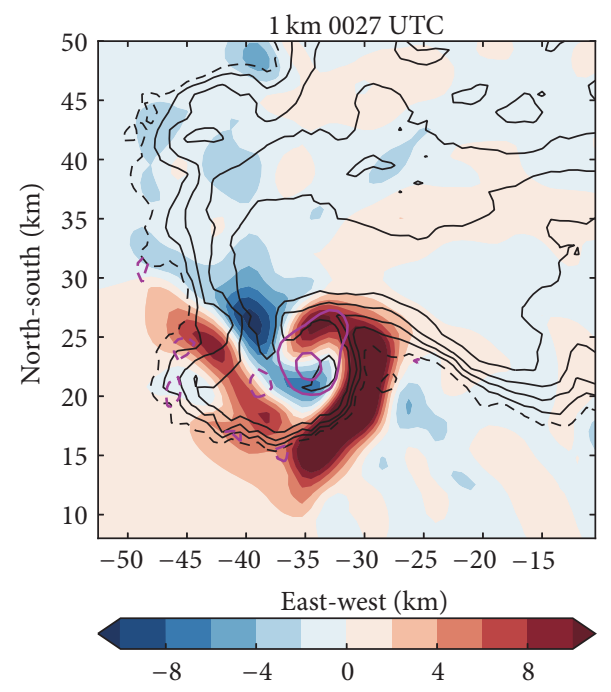

(b)

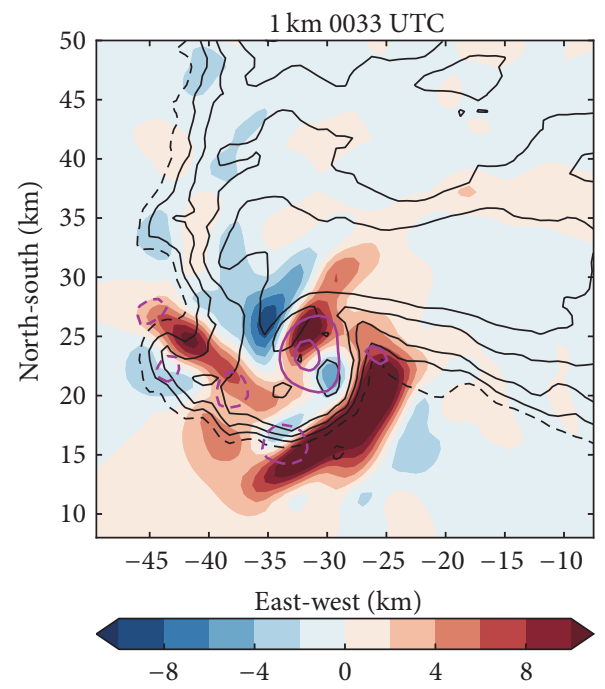

(d)

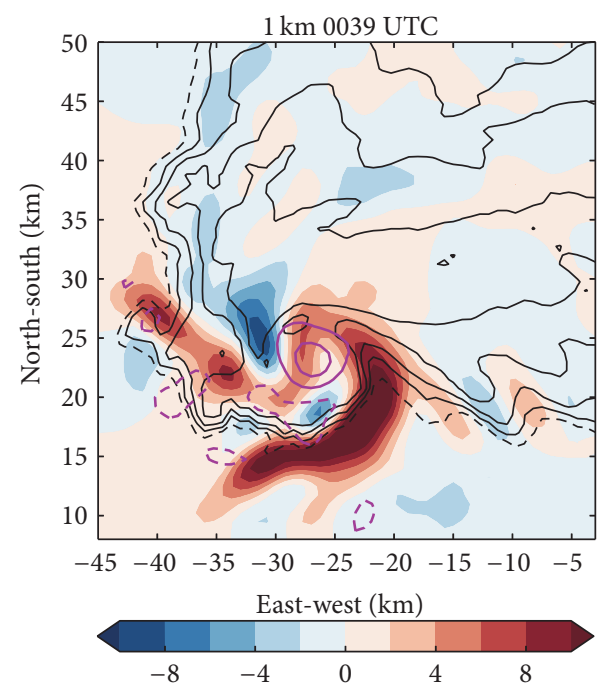

(f)

FIgURE 5: Same as Figure 3, except for during the mature stage. 
of updraft at the leading edge of the SRFGF (Figure 5(a), $x=$ $-44, y=25$ ). This updraft region was consistently located behind the main gust front and leading reflectivity gradient (Figures 5(b), 5(d), and 5(f)). This western updraft also remained discrete from the primary updraft, even its trailing section, at middle and upper levels. At low levels, this new updraft region limited further penetration of RFD air into the mesocyclone (Figures 5(d) and 5(f)). Furthermore, at middle levels, the western updraft zone blocked the entrainment of dry environmental air from the southwest into the RFD, which would also likely have limited the strength of the surface cold pool.

The downdraft structure also changed dramatically between the organizing and mature stage of the mesocyclone. In the early organizing stage, the RFD at one $\mathrm{km}$ altitude was elongated and located along the back edge of the radar reflectivity gradient (Figure 3(b)). During the mature stage, the near-surface RFD became more compact and was continually collocated with the highest radar reflectivity just northwest of the mesocyclone (Figures 5(d) and 5(f)). Even though the low-level downdraft intensified, or at least maintained its strength over the period, the volume somewhat decreased with time (Figures 6(a), 6(c), and 6(e)). Meanwhile, the forward flank downdraft increased in size and intensity at middle-to-upper levels (Figures 6(a), 6(c), and 6(e)) but remained weak near the surface, consistent with the lack of a well-defined surface boundary along the southern edge of the forward flank of the storm.

The occlusion downdraft evolved throughout the mature stage as well. Initially, the occlusion downdraft was embedded near the center of the mesocyclone on the south-southwest side of the circulation (Figure 5(b), $x=-35, y=21$ ). With time, the occlusion downdraft shifted outward and rotated cyclonically around the circulation and was located on its southern periphery by 0039 UTC (Figure 5(f), $x=-26$, $y=16)$.

In addition to the occlusion downdraft, there was evidence of a distinct occlusion updraft, particularly near the surface at 0033 UTC (Figure 5(d), $x=-32, y=25$ ). The occlusion updraft was less obvious at the beginning of the mature stage (0027 UTC) as it appeared to be a northern appendage of the primary updraft zone. With time, however, the core of the primary updraft (denoted by its maxima) shifted clockwise relative to the mesocyclone, producing better separation between the primary and occlusion updrafts. By 0039 UTC, the occlusion updraft filled the low-level mesocyclone, creating a temporary reprieve from the divided updraft/downdraft structure more commonly observed during the mature stage of a mesocyclone [17].

4.2.3. Evolution of Vertical Vorticity. During the mature stage of its lifecycle, the vertical structure of the second mesocyclone continued transitioning from being tapered in diameter with height to being nearly uniform in diameter with height (Figure 6(b)). This transition was likely associated with stretching of vertical vorticity by the vertical gradients in vertical motion as the mesocyclone went from a divided vertical velocity structure to being dominated by the occlusion updraft that intensified with height (Figure 6).
Interestingly, the deep mesocyclone continued to intensify even though it was horizontally displaced from the primary updraft. The vertical velocity gradients in the primary updraft also amplified vertical vorticity, but this updraft shear region vorticity structure was well separated from the vorticity that connected to the low-level mesocyclone (Figures 6(b), 6(d), and $6(f))$. As the updraft core shifted clockwise relative to the mesocyclone vortex, the updraft shear region vorticity also shifted clockwise and became fully disconnected from the mesocyclone vortex by 0039 UTC.

\subsection{Occlusion Stage of the Second Mesocyclone: 0042-0052 UTC}

4.3.1. Low-Level Boundaries. The onset of the occlusion of the second mesocyclone after 0042 UTC was marked by a southeastward surge in the RFGF (cf. Figures 3(a) and 3(c) with Figures 7(a) and 7(c)). The SRFGF remained attached to, but expanded eastward to encompass, the occluding circulation. In a storm-relative sense, the circulation moved west-northwestward. At 0052 UTC, the primary RFGF and SRFGFs were nearly colocated at one $\mathrm{km}$ altitude (Figures $7(\mathrm{e})$ and $7(\mathrm{f})$ ), as the southerly environmental air flowed over the shallow western end of the primary RFGF and converged with midlevel air within the storm that was flowing southwestward around the western side of the mesocyclone.

The FFCB also remained attached to the occluding mesocyclone. However, the southern end of the boundary rotated from the northwest to the northeast side of the mesocyclone in association with the southeast low-level environmental inflow being cut-off from the circulation (Figure $7(\mathrm{e})$ ).

4.3.2. Vertical Draft Evolution. Unlike the mature stage, where the RFD stayed along the northwest exterior of the mesocyclone, during the occluding stage the primary RFD circulated around the western and southern sides of the mesocyclone (Figures 7(b) and 7(d)) and merged with a deep, strong occlusion downdraft (Figures 8(a), 8(c), and 8(e)) within the southern edge of the mesocyclone (Figure $7(\mathrm{f})$ ). The relative strengths of the RFD and occlusion downdrafts also switched between the mature and occlusion stages of the mesocyclone. During the occlusion period the occlusion downdraft (Figure $7(\mathrm{f}), x=-20, y=24$ ) was significantly stronger than the RFD (Figure $7(\mathrm{f}), x=-23, y=$ 27). The relative strength of the occlusion downdraft and the limited southward extent of the SRFGF suggest that the occlusion downdraft played a more significant role in the occlusion of the mesocyclone compared to the RFD. Nevertheless, the cyclonic rotation of the RFD air, reinforced by the strong occlusion downdraft, was responsible for the eastern expansion of the SRFGF and the southeastern surge in the primary RFGF. The RFGF surge also coincided with expansion of the high reflectivity core on the southwestern side of the hook echo. This increase in reflectivity was a dramatic change from the narrow reflectivity core on the west side of the circulation that had been previously present for over 30 minutes.

During the occlusion process, the vertical velocity structure within the mesocyclone transitioned from being 


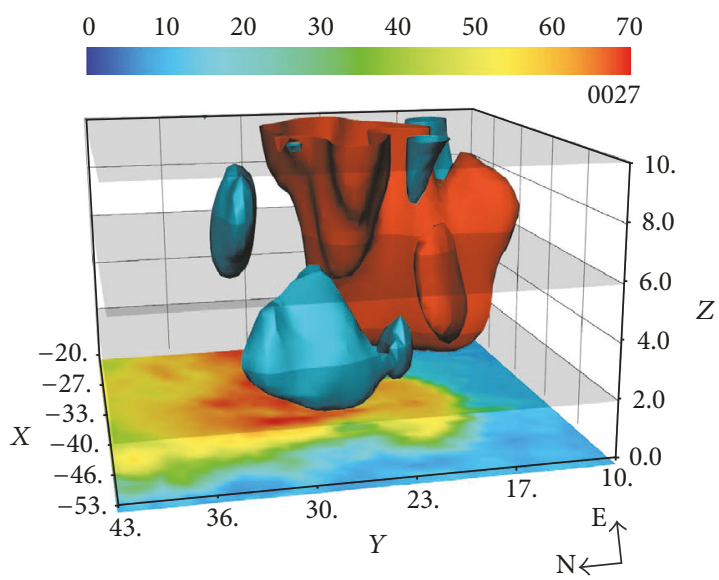

(a)

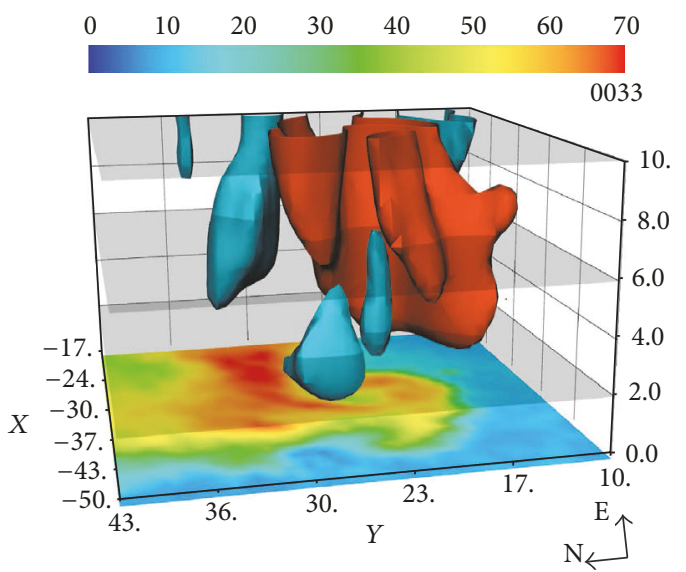

(c)

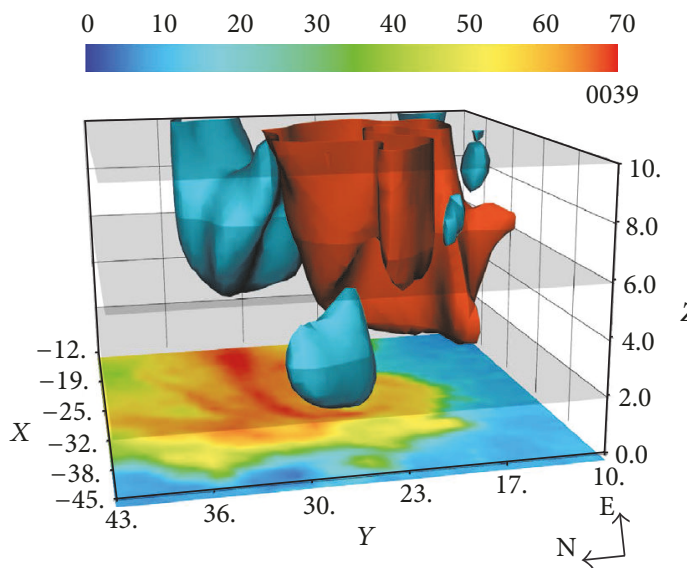

(e)
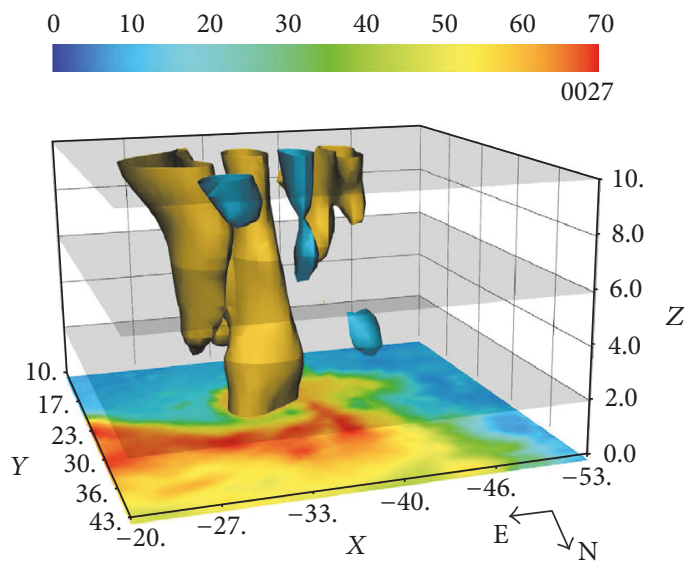

(b)

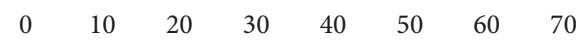

0033

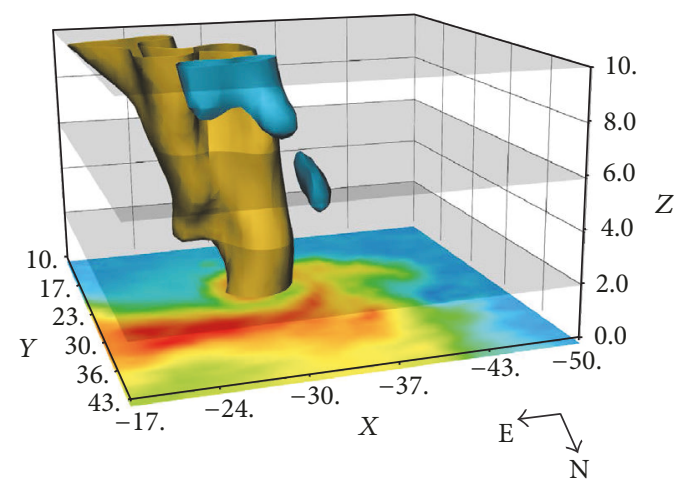

(d)
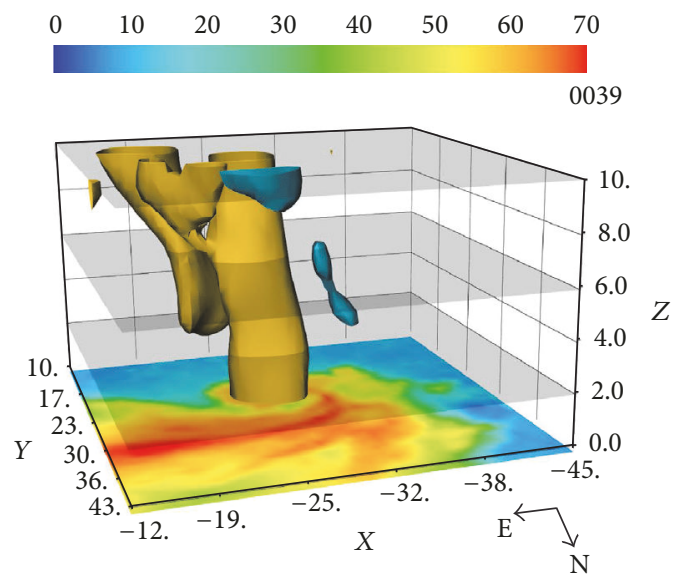

(f)

FIGURE 6: Same as Figure 4, except for during the mature stage.

mostly upward motion back to a more divided structure, consistent with the Lemon and Doswell III [17] conceptual model. The core of the updraft on the northern side of the mesocyclone remained separated from the strong upward motion in the primary updraft region, which continued to shift its core clockwise relative to the mesocyclone. As the RFGF moved away from the mesocyclone, the low-level convergence weakened and the low-level updrafts diminished (cf. Figures 3(f), 5(d), and $7(f))$.

4.3.3. Evolution of Vertical Vorticity. Initially, the mesocyclone itself was collocated with the occlusion updraft (Figure 7(b), $x=-25, y=24$ ) over a deep layer and therefore continued to intensify. Additionally, as sinking motion wrapped around the low-level circulation, the mesocyclone 


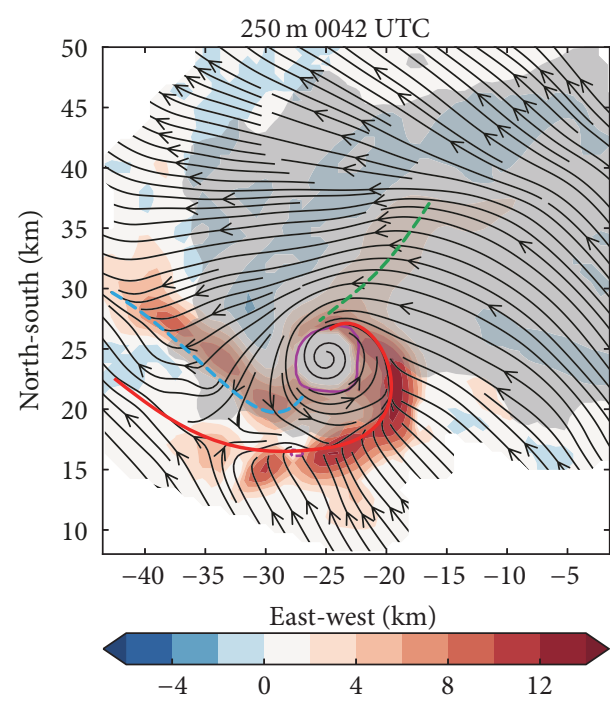

(a)

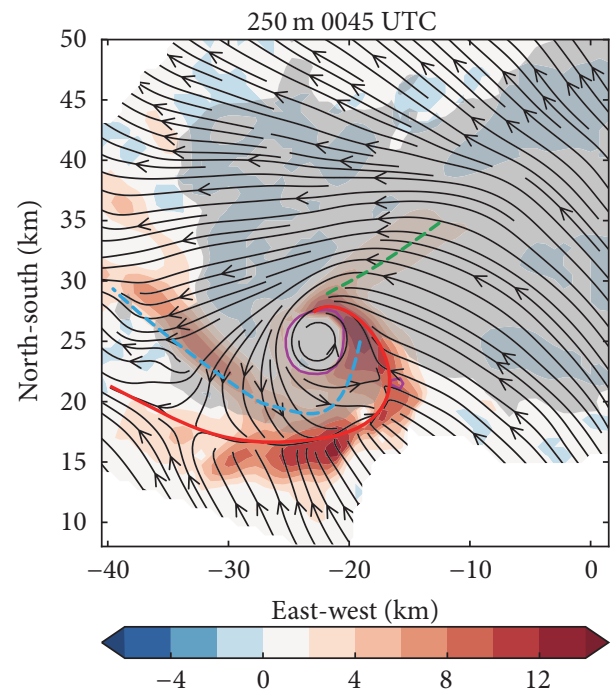

(c)

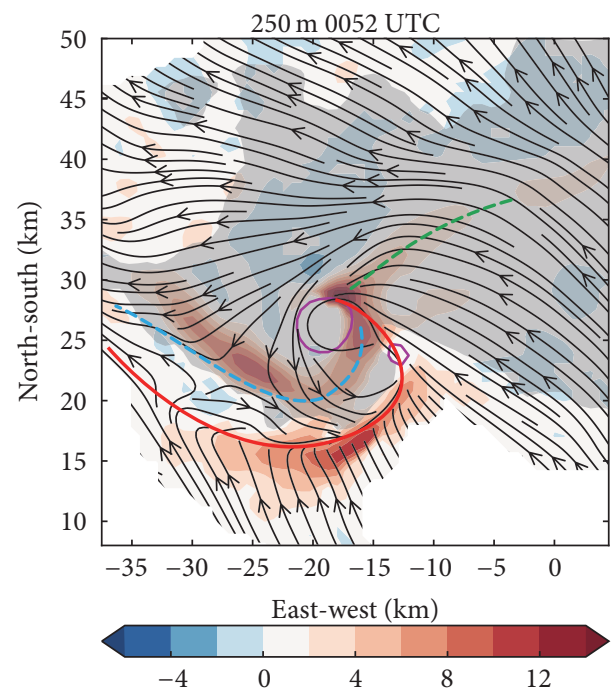

(e)

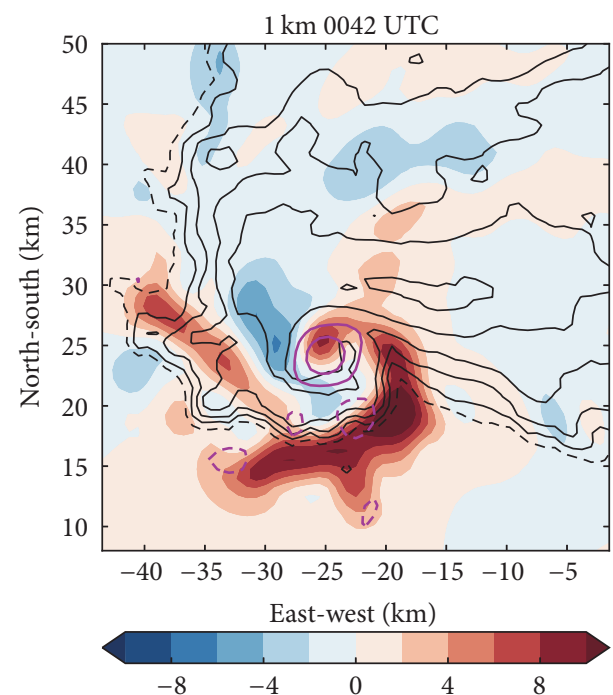

(b)

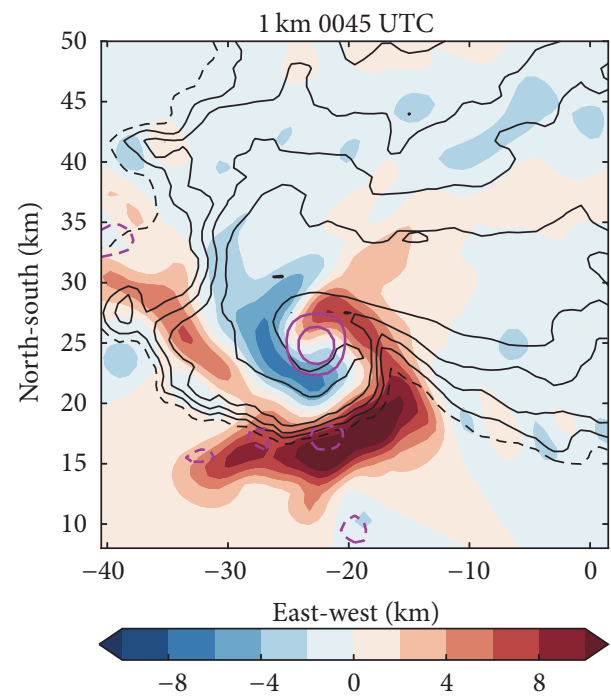

(d)

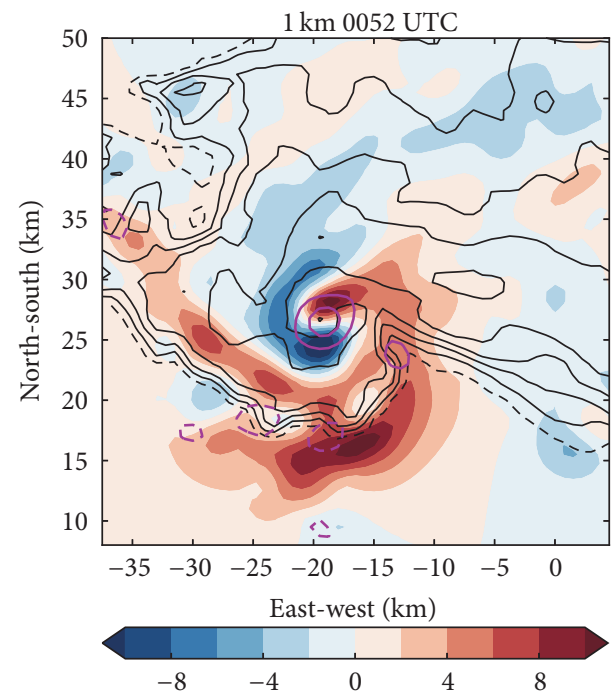

(f)

FIgURE 7: Same as Figure 3, except for during the occlusion stage. 


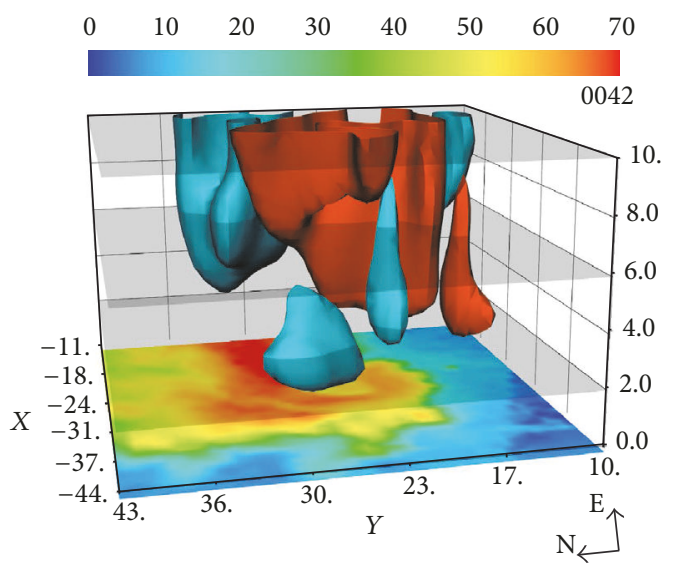

(a)

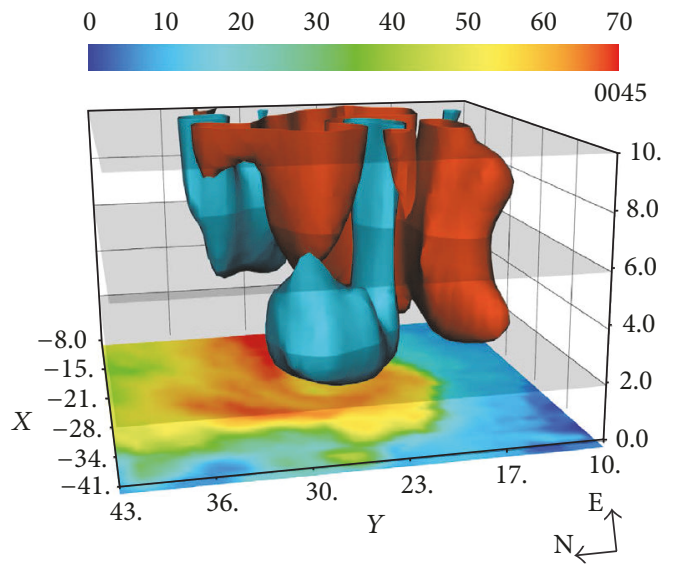

(c)

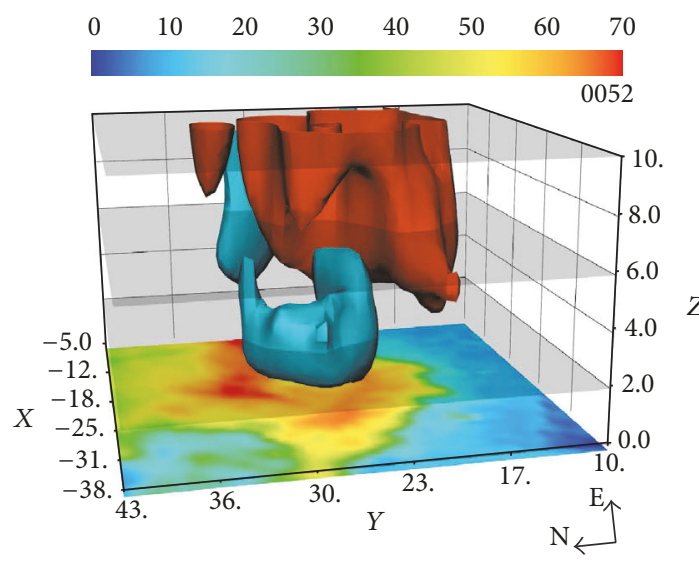

(e)

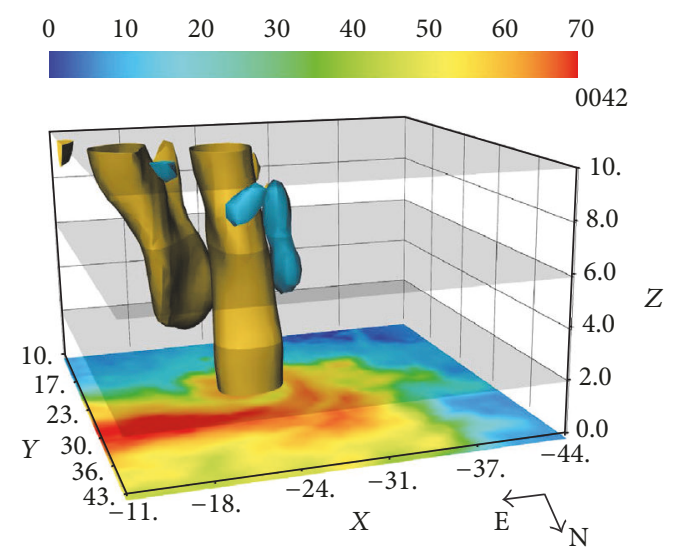

(b)
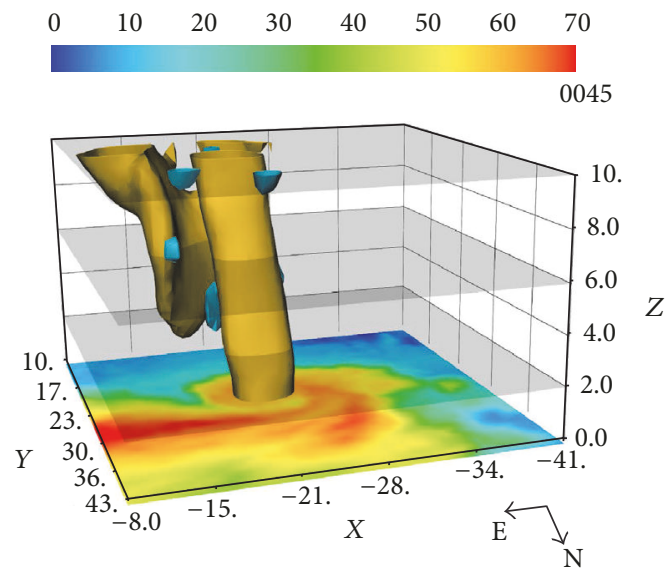

(d)
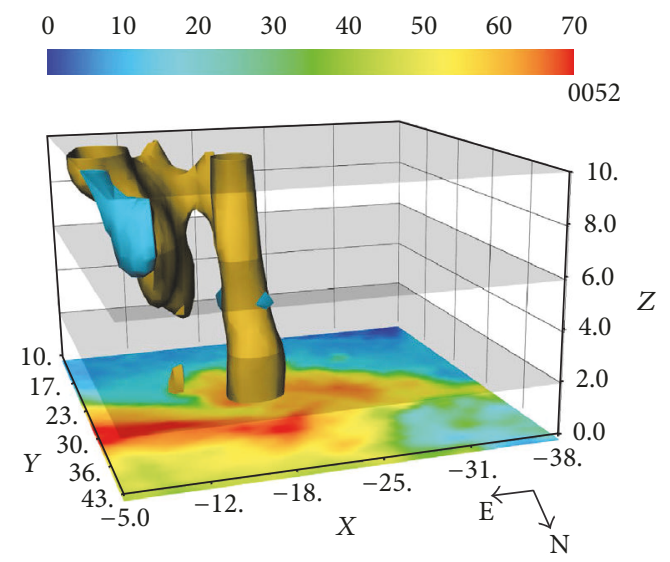

(f)

FIGURE 8: Same as Figure 4, except for during the occlusion stage.

became more symmetric and decreased in scale. Meanwhile, at middle levels, the mesocyclone vortex continued to separate from the updraft shear region vorticity (Figures $8(\mathrm{~b}), 8(\mathrm{~d})$, and $8(\mathrm{f})$ ), with a region of anticyclonic vorticity developing between the two positive vorticity regions at 0045 UTC (Figure 8(d)). At 0052 UTC, a tornado developed in association with the occluding second mesocyclone while a small but new and distinct vorticity maximum can be seen at low levels (Figure 7(f), $x=-12, y=23$; Figure 8(f)). It developed beneath the northern tip of the updraft shear region vorticity and later elongated and intensified into the third mesocyclone. 


\subsection{Development Stage of the Third Mesocyclone: 0058-0108 UTC}

4.4.1. Low-Level Boundaries. As the mesocyclone continued to occlude, the primary RFGF pushed east (Figures 9(a), 9(c), and 9(e)) and became detached from the circulation around 0105 UTC, or about 15 minutes after the occlusion process began. The FFCB also detached from the occluding mesocyclone but remained connected to the primary RFGF at the location of the developing region of cyclonic vorticity (Figures 9(c) and 9(e)). In contrast to the previous mesocyclone cycle, the FFCB was manifested as a more pronounced wind shift boundary even though the asymptotic contraction rate was similar in magnitude to previous times. On the other hand, the asymptotic contraction rate associated with the SRFGF diminished. Near the end of the redevelopment stage, the asymptotic contraction rate on the east side of the mesocyclone marked the eastern extent of the old SRFGF and the western extent of a new SRFGF.

4.4.2. Vertical Draft Evolution. An elongated reflectivity core formed between the occluding circulation and the primary updraft (Figure 9(d), $x=-9, y=23$ ) as the hook echo region underwent significant changes, particularly along the primary RFGF where two inflow notches were observed. The main updraft was in the southeastern inflow notch where the new mesocyclone developed (Figure 9(d), $x=$ $-4, y=22$ ) while a secondary updraft formed in the smaller inflow notch in the RFGF south of the occluding mesocyclone (Figure 9(f), $x=-13, y=17$ ) and extended nearly to the occluding mesocyclone in a fashion similar to the previous cycle (Figure 3(b)). Near-surface streamlines (Figure 9) indicate that the southeastern inflow remained attached to the occluding circulation until about 0105 UTC when the winds switched from east-northeasterly to northnortheasterly.

Despite the surging outflow relative to the occluding circulation, the RFGF remained underneath the midlevel updraft (Figure 10), similar to the 8 June 1995 McLean, TX, storm of Dowell and Bluestein [6]. Perhaps the favorable balance between the relative strengths of the inflow and outflow allowed the new mesocyclone to spin up quickly.

Interestingly, the southwestern band of updraft that had been prevalent for more than 30 minutes during the mature and occluding stage of the second mesocyclone evolution had weakened considerably and no longer separated the old SRFGF from the primary RFGF.

The strongest downdraft in the rear-flank region was associated with the occluding mesocyclone and stayed mainly on the western edge of the weakening circulation, though a new occlusion downdraft formed in the southern part of the occluding mesocyclone at 0108 UTC (Figure 9(f), $x=-14$, $y=26$ ). Additionally, downward motion to the northeast of the occluding mesocyclone was observed throughout this period (cf. Figure 9(f), $x=-5, y=32$ ). This northeastern RFD aided the development of the new SRFGF observed in the 0108 UTC asymptotic contraction rate analysis. The increased northerly component of flow on the eastern side of the occluding mesocyclone coincided with development of this new RFD. This may imply that the pressure gradient force associated with the occluding mesocyclone sufficiently weakened in the elongated inflow region to allow the wind to align with the pressure gradient force from the new RFD.

4.4.3. Evolution of Vertical Vorticity. The occluded portion of the RFGF was at the center of the so-called vorticity-rich region in the Burgess et al. [5] conceptual model. This region developed underneath the northern tip of the updraft shear region vorticity (Figure 9 (f)) and, as the vorticity region of the third mesocyclone elongated, extended upward in height (Figure $11(\mathrm{~d}), x=-5, y=24)$ producing a deep column of vorticity (Figure 11(d)) associated with stretching in the primary updraft after 0058 UTC (Figures 9(d) and 9(f); Figures 11(a), 11(c), and 11(e)). As with the organizing stage of the previous low-level mesocyclone, a cyclonic/anticyclonic vorticity couplet formed on the edge of the elongated hook echo with vortex arches [67] connecting the two. The tornadic, occluding, second mesocyclone maintained a deep column of vorticity throughout this time (Figures 11(b), 11(d), and 11(f)).

\subsection{Organizing Stage of the Third Mesocyclone: 0112-0118 UTC}

4.5.1. Low-Level Boundaries. The new SRFGF pushed south during the organizing stage of the third mesocyclone and eventually wrapped around the southern end of the new mesocyclone, which had developed rapidly from the elongated vorticity region (Figures 12(a), 12(c), and 12(e)). The SRFGF also pushed westward with time, appearing to undercut the occluding circulation and aiding in the demise of its associated tornado (Figures 12(c) and 12(e)). By the end of the new organizing stage, the new SRFGF was nearly indistinguishable with the old SRFGF, which had merged with the primary RFGF on the western side of the domain (Figure 9(e)).

4.5.2. Vertical Draft Evolution. The westward SRFGF surge was coincident with strengthening of the new RFD and the expansion of the hook echo (Figures 12(d) and 12(f)). In many ways, during 0112-0118 UTC the old RFD and occluding mesocyclone were similar in structure to the old RFD and occluding circulation during the previous organizing stage (2358-0022 UTC; cf. Figure 3 with Figure 12). The strongest downdraft was initially with the older mesocyclone. However, as that circulation dissipated, the old RFD weakened with the remaining subsidence mostly along the western edge of the hook echo. Even as the primary RFD strengthened and shifted south (Figure 12(b), $x=-5, y=32$, Figure 12(f), $x=-1, y=27)$, the downdraft was shallow and was located below rising motion at middle levels (Figures 13(a), 13(c), and $13(\mathrm{e}))$, similar to the updraft structure observed in the previous cycle.

The primary low-level updraft region of the new mesocyclone consisted of discrete updraft cores, which were just starting to form a more contiguous band, similar to the structure at 2358 UTC. As before, the southwestern updraft 


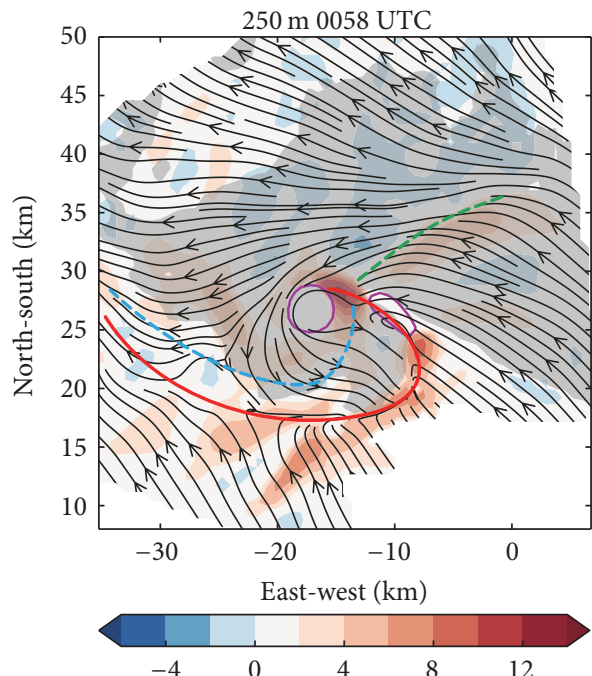

(a)

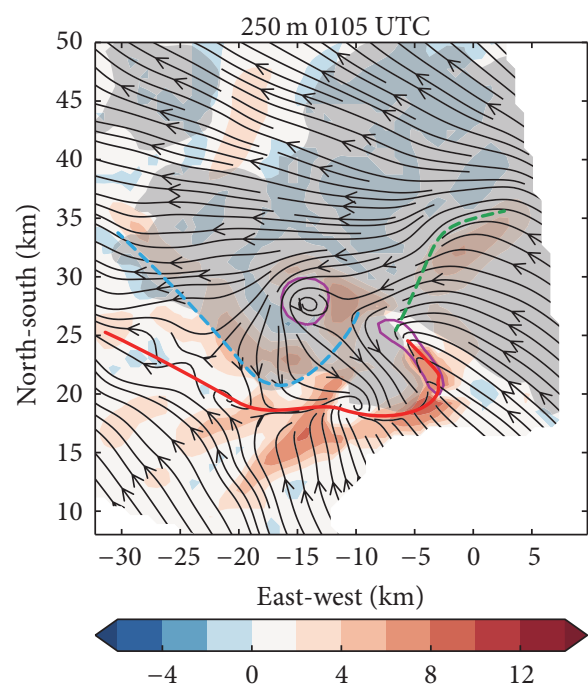

(c)

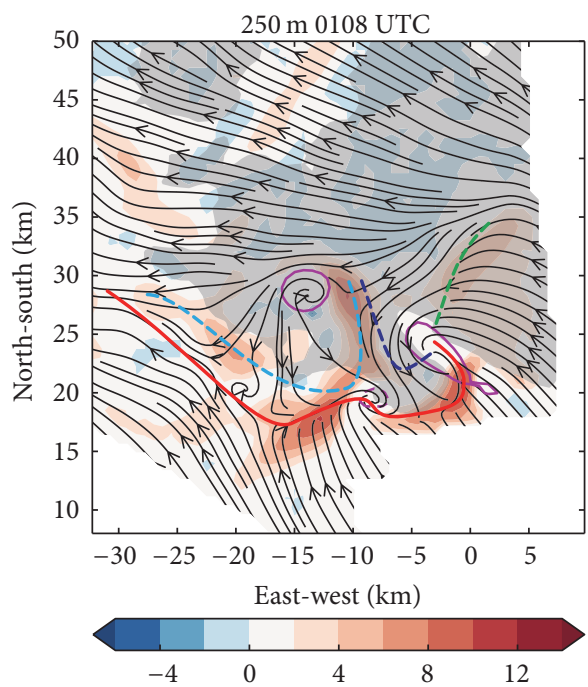

(e)

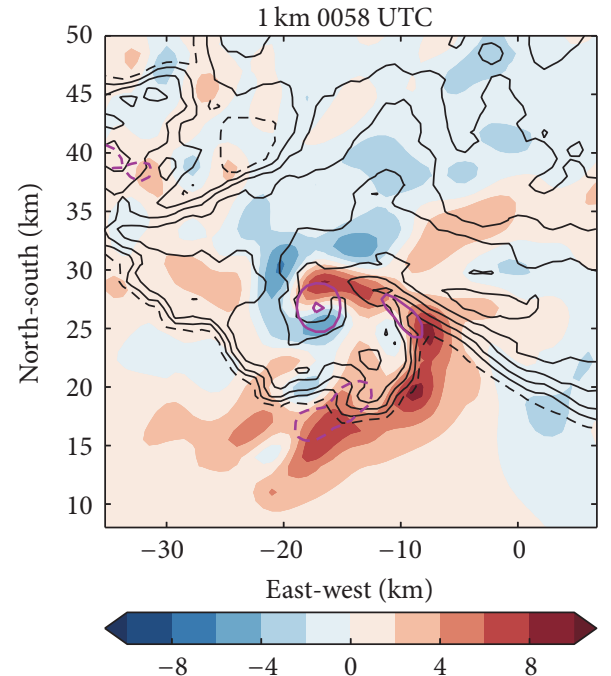

(b)

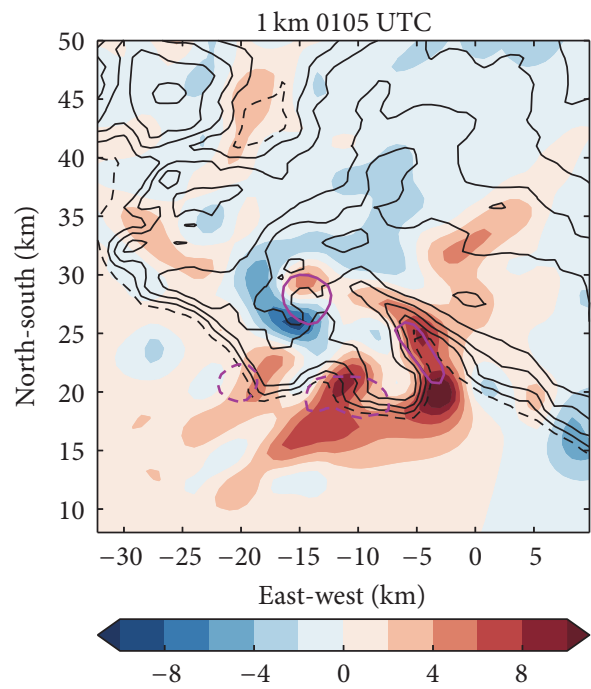

(d)

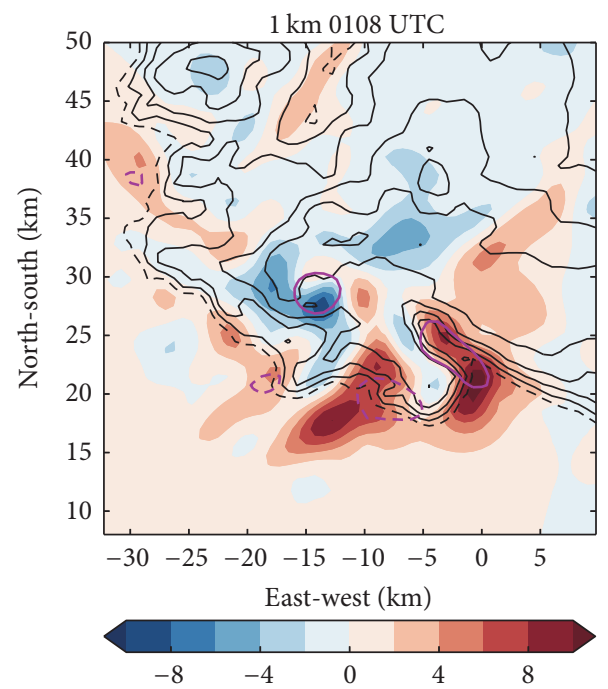

(f)

FIGURE 9: Same as Figure 3, except for during the development stage of the third mesocyclone. 


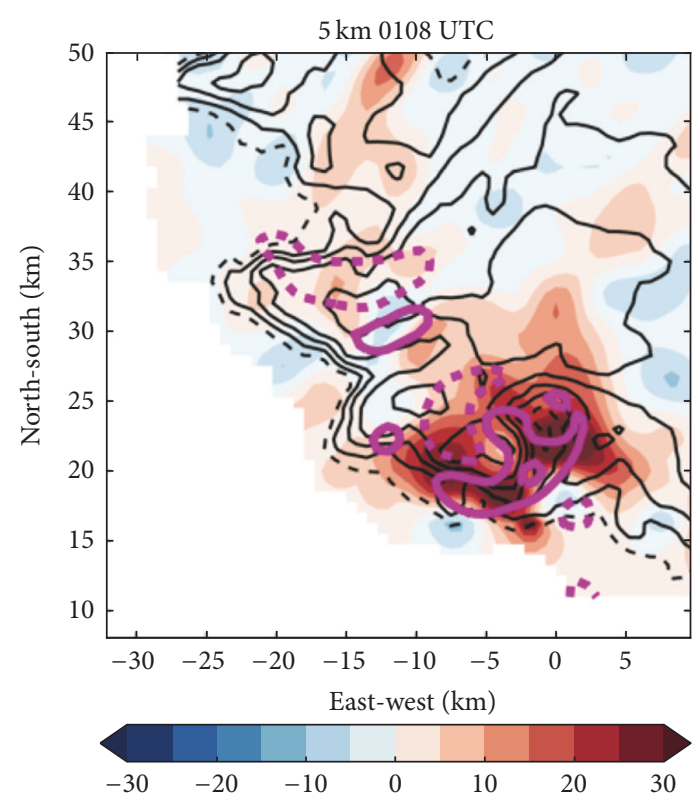

Figure 10: Horizontal cross-sections at $5 \mathrm{~km}$ altitude for the 0108 UTC analysis of vertical velocity (in $\mathrm{m} \mathrm{s}^{-1}$, according to the color scale), radar reflectivity greater than $20 \mathrm{dBZ}$ (contoured every $10 \mathrm{~dB}$ with $20 \mathrm{dBZ}$ dashed and higher values solid). Vertical vorticity maxima are annotated in magenta at -5 (dashed), 10 , and 30 (solid) $\left(\times 10^{-3} \mathrm{~s}^{-1}\right)$.

band was not present during the organizing stage of the new mesocyclone. However, the structure of the new SRFGF and associated asymptotic contraction rate analysis suggests that a new southwestern band would form during the mature stage of the new cycle, just as was observed in the prior mature stage cycle.

4.5.3. Evolution of Vertical Vorticity. The occluding, tornadic mesocyclone (Figure 13(b), $x=-14, y=30$ ) continued to move rearward in time and became tilted with height towards north-northeast (Figure 13(d)). The size of the occluding circulation had decreased at all levels. By 0115, the midlevel vorticity maximum had vanished and only a small maximum near the surface remained. Observations suggest that the cyclonic tornado dissipated at approximately 0115 UTC.

Meanwhile, the first of two anticyclonic tornadoes dissipated at 0112 UTC and the second one formed around 0115 UTC. The three-dimensional vorticity analysis reveals how the original anticyclonic vorticity column grew a perturbation that split into two separate anticyclonic circulations at 0115 UTC (Figures 13(b) and 13(d)). The first anticyclonic tornado dissipated as the second tornado formed. The second tornado was associated with the northwestern circulation.

The organizing new low-level mesocyclone, which had benefited from stretching in the primary updraft during its formative stage, was starting to become a distinct vorticity column as it shifted southwestward relatively to the updraft shear region vorticity between 0115 and 0118 UTC (Figures 13(d) and 13(f)). This break between the vorticity column associated with the low-level mesocyclone and the deep vorticity column associated with the primary updraft had apparently already occurred prior to 2358 UTC in the previous mesocyclone cycle.

\section{Discussion}

Figure 14 summarizes the evolution of low-level boundaries diagnosed from the asymptotic contraction rate analysis from the end of the first mesocyclone to the organizing stages of the third mesocyclone. Identifiable boundaries were plotted relative to low-level downdrafts, reflectivity, and midlevel vorticity structures. Unlike the previous near-surface boundary illustrations, a distinction was made between boundaries with distinct wind shifts (solid) and solely persistent regions of enhanced contraction rate that became wind shift boundaries in the future (dashed).

The organizing mesocyclone stage was observed for two different mesocyclone cycles: at 2358 UTC (Figure 14(a)) and 0112 UTC (Figure 14(k)). Multiple hook echoes were present during each cycle, one associated with the occluding circulation and the other associated with the new circulation. The SRFGFs associated with the occluding circulations both retreated to the northwest side of the storm and extended southward towards the primary gust front during both cycles.

While the occluding circulation was significantly stronger during the later cycle, the most impactful storm-scale difference at the two times was the deep, anticyclonic vorticity on the west side of the hook echo at 0112 UTC (Figure 15(b), $x=-8, y=24)$ compared to the weak cyclonic vorticity observed at 2358 UTC (Figure 15(a), $x=-58, y=$ 23). This difference is consistent with the development of anticyclonic tornadoes during the organizing stage of the third mesocyclone and the lack of anticyclonic tornadoes during the organizing stage of the second mesocyclone. The first of the two substantial anticyclonic tornadoes formed in the southern anticyclonic region at 0102 UTC and lasted for approximately ten minutes. Both tornadoes were associated with deep anticyclonic vorticity columns evident at 0112 UTC (Figure 13(b)) that persisted beyond the lifecycle of the tornadoes (Figure 13(d)). The mesoanticyclone associated with the anticyclonic tornadoes during the third cycle caused the RFGF to bend northward and potentially slowed the southward advances of the SRFGFs associated with the occluding and developing circulations.

By the end of the organizing stage of the second mesocyclone (0016 UTC; Figure 14(b)), divergence from the old RFD had reinforced a kinematic boundary extending north from the circulation and the SRFGF to the west. Interestingly, the implied intersection of the three boundaries remained on the northwest side of the circulation as the vortex expanded to the southwest and increased in asymmetry. In contrast, the triple point was on the northeast side of the circulation during the organizing stage of the third mesocyclone, between 0112 and 0118 UTC (Figures 14(k) and 14(1)). This difference was due to the new RFD that formed to the northeast of the developing mesocyclone during the organizing stage of the third mesocyclone. A similarly strong new RFD was lacking in the prior organizing stage.

The southern part of the primary RFGF did not push farther southward until the SRFGF progressed around the 


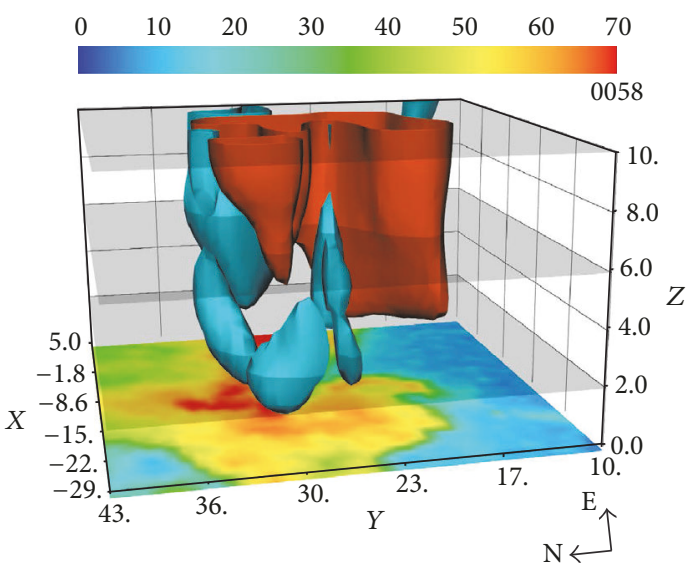

(a)

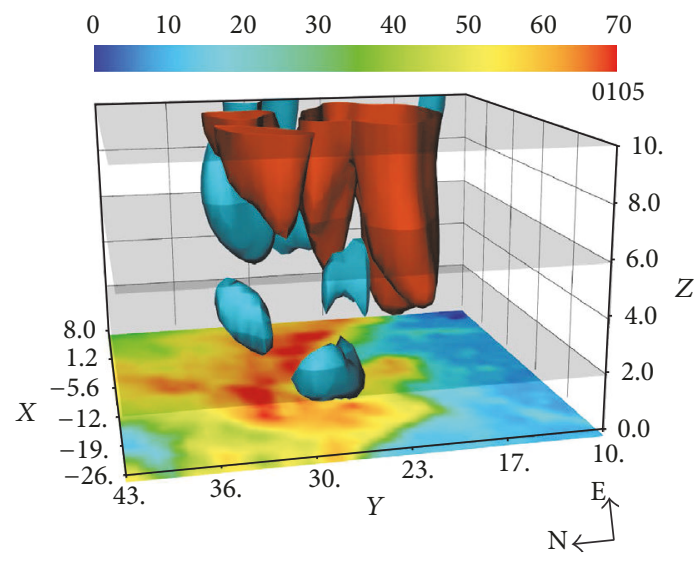

(c)

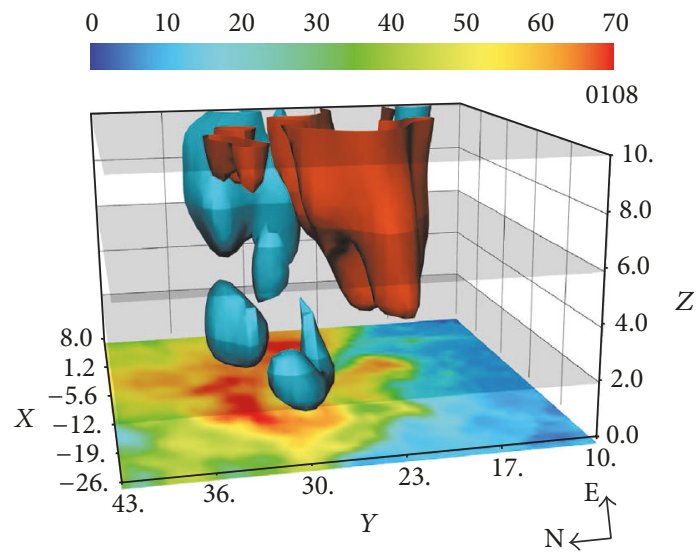

(e)

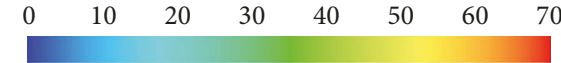

0058

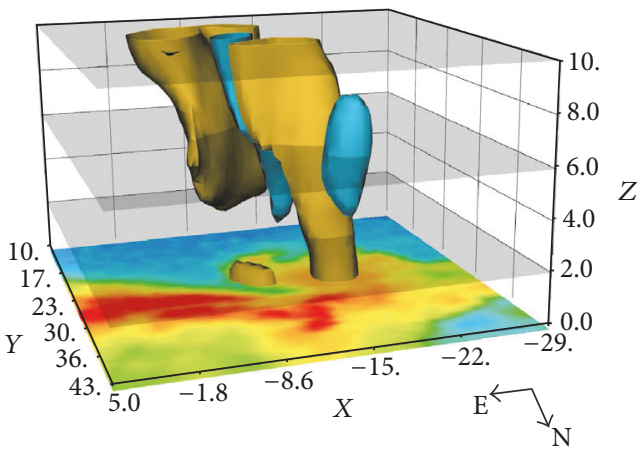

(b)

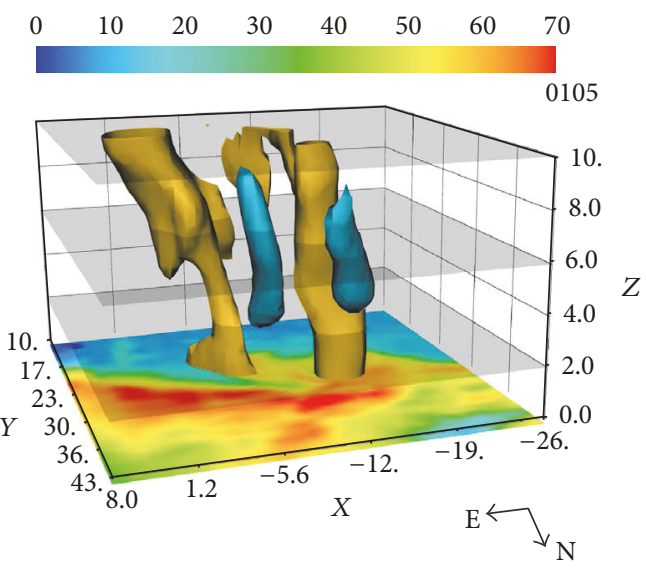

(d)

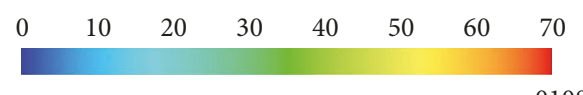

0108

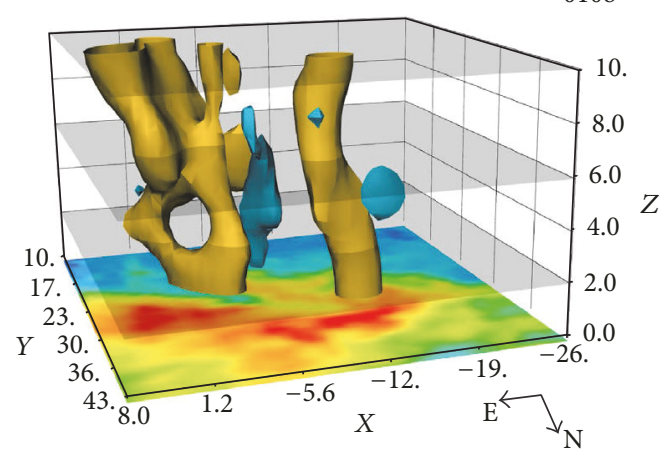

(f)

FIGURE 11: Same as Figure 4, except for during the development stage of the third mesocyclone.

southern edge of the second mesocyclone circulation (Figures $14(\mathrm{e})-14(\mathrm{~g}))$. Despite the RFD intensification, convergence along the FFCB (Figure 5(a)) did not noticeably change, suggesting that the increased outflow from the RFD was directed mainly westward towards the SRFGF. Indeed, preceding the onset of the occlusion stage of the second mesocyclone, the SRFGF pushed westward (Figure 14(f), 0045 UTC) and a deep mesoanticyclone rotated from being on the southwest side of the cyclonic mesocyclone to being the southeast side (Figure 16). The resulting change in largescale deformation was reflected in the low-level reflectivity, which rapidly expanded on the western side of the hook echo.

The near-surface boundary evolution during the occlusion stage of the second mesocyclone was consistent with the conceptual models of Burgess et al. [5] and Dowell and Bluestein $[6,18]$ as well as the high-resolution observations of 


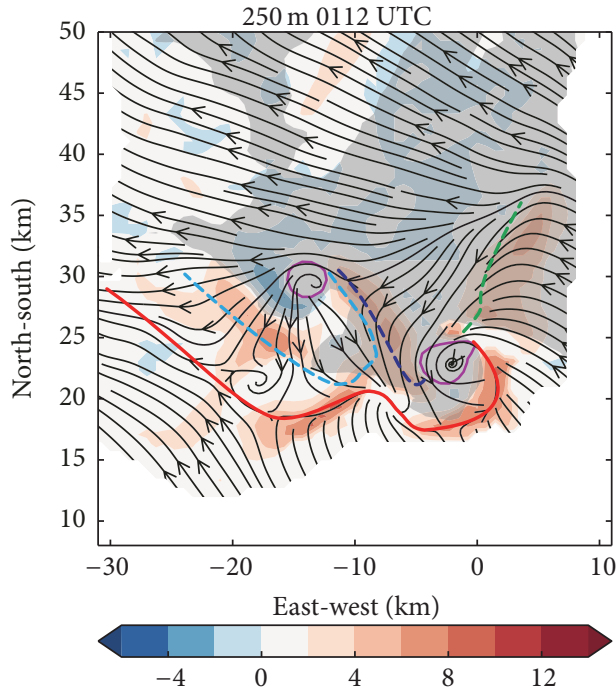

(a)

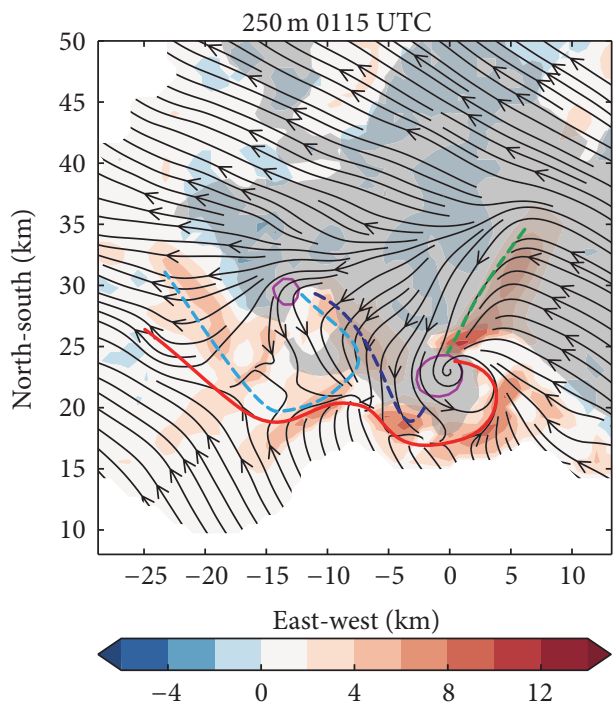

(c)

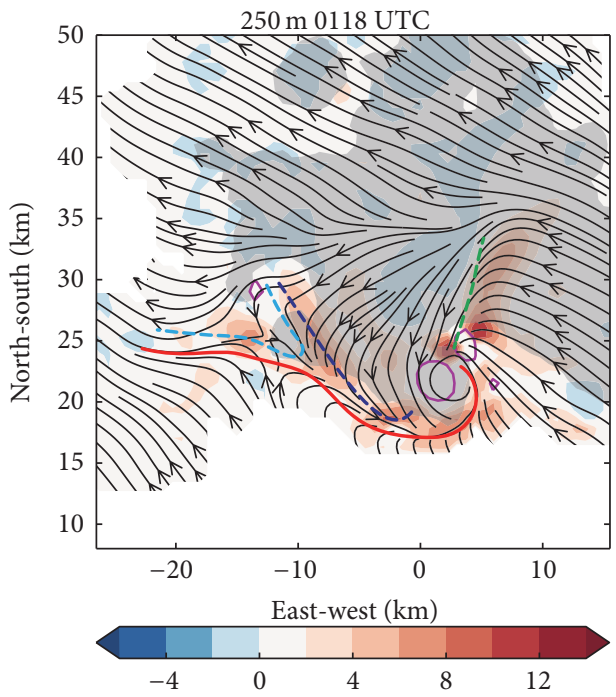

(e)

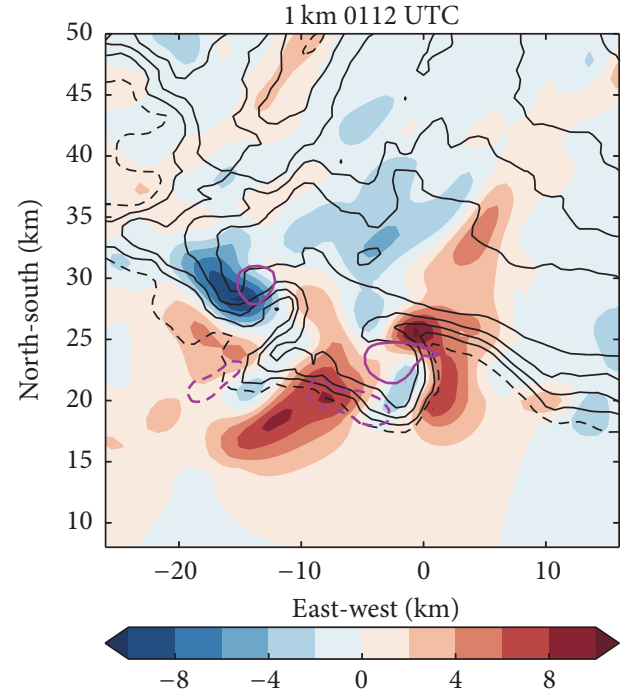

(b)

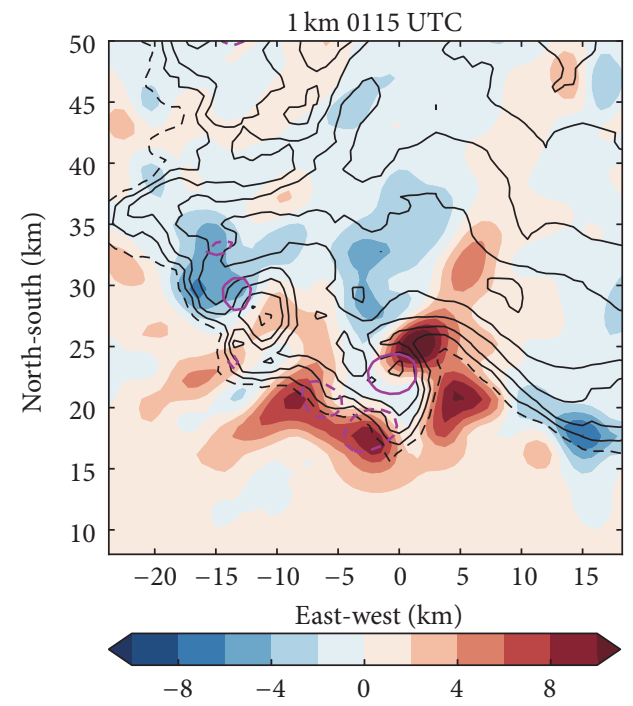

(d)

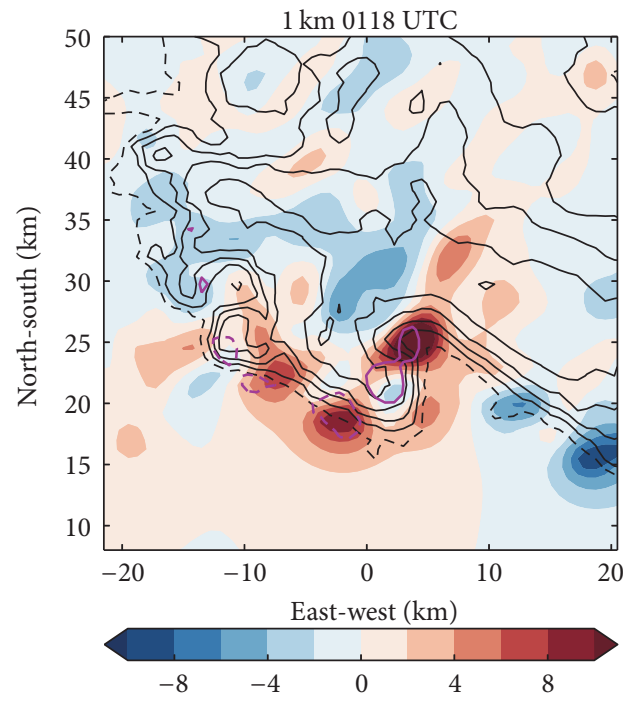

(f)

FIGURE 12: Same as Figure 3, except for during the organizing stage of the third mesocyclone. 


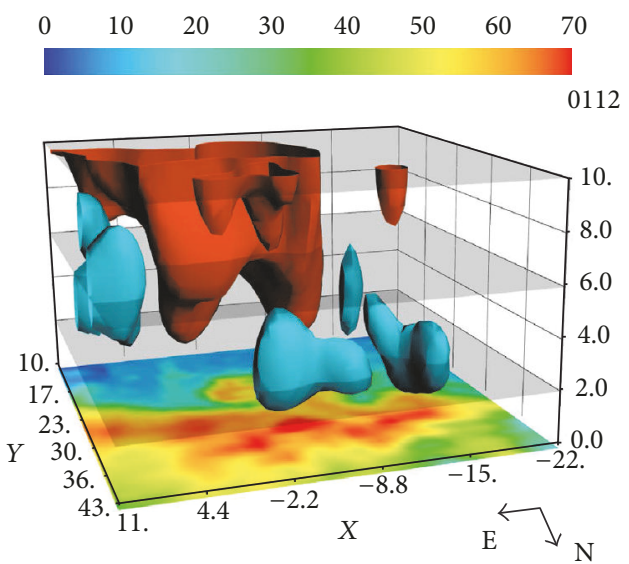

(a)

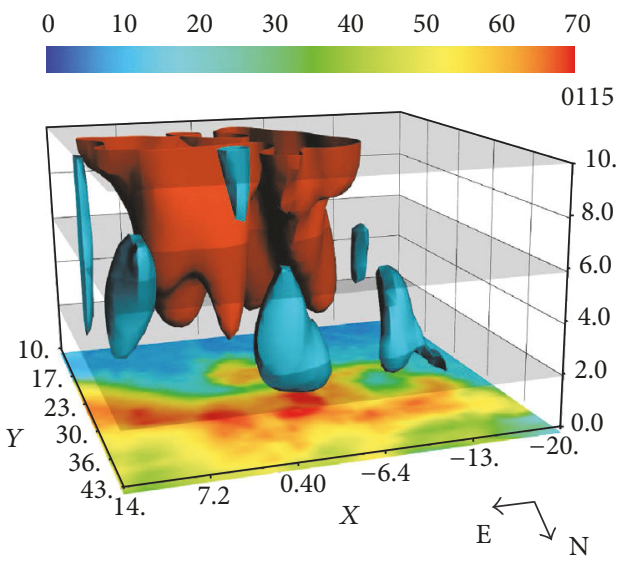

(c)

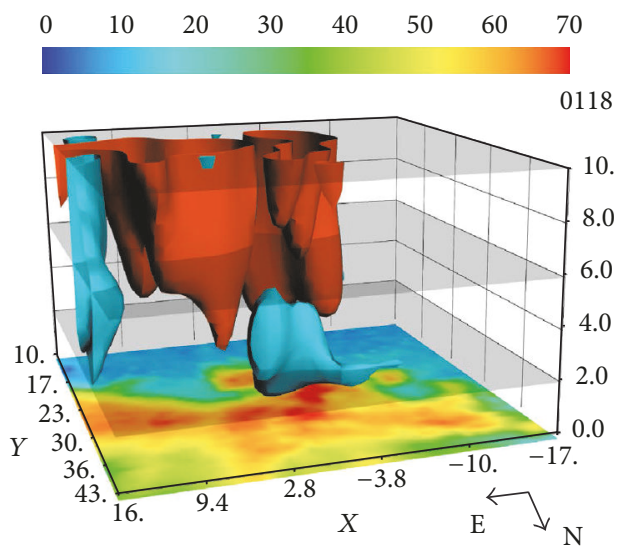

(e)

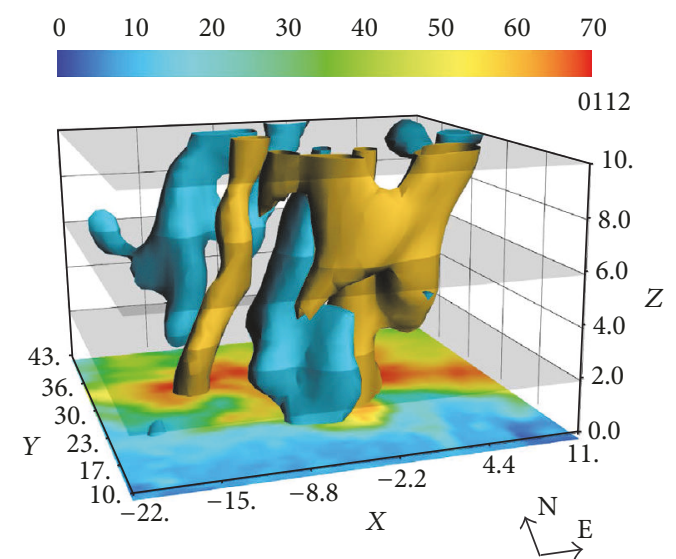

(b)

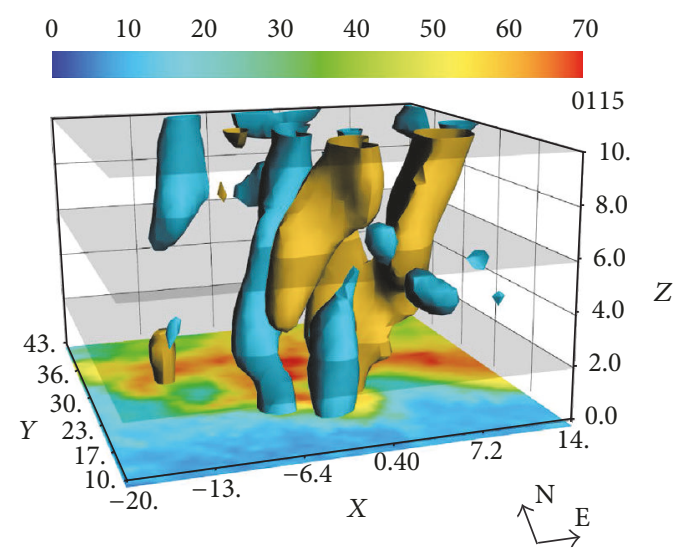

(d)
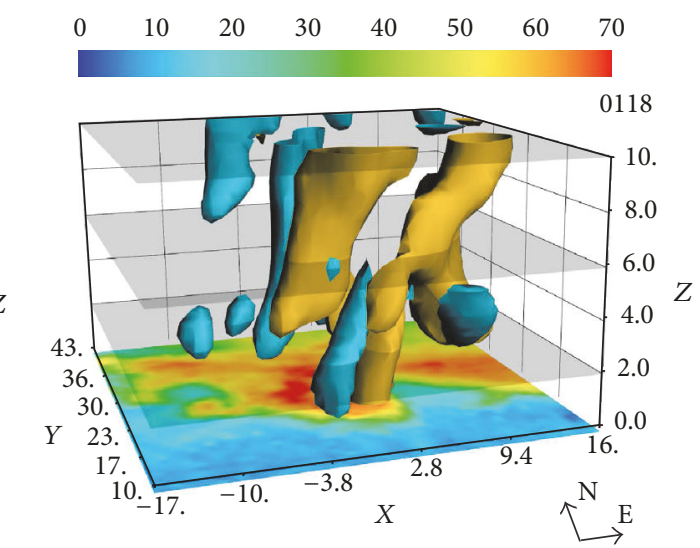

(f)

FIGURE 13: Same as Figure 4, except during the organizing stage of the third mesocyclone and the orientation has changed. The vertical velocity (panels (a), (c), and (e)) is from the perspective of looking at the storm from roughly the north. The vorticity (panels (b), (d), (f)) is from the perspective of looking at the storm from roughly the south.

Marquis et al. [28]. The onset of the occlusion stage after 0045 UTC was marked by a southeastward surge in the primary RFGF, while the SRFGF remained with the occluding circulation. The occluding tornadic mesocyclone also remained connected to the occluded portion of the primary RFGF until northerly winds from the RFD pushed the occluded RFGF boundary southward. Concurrently, convergence along the
FFCB extended well to the northeast and then advanced eastward along the primary RFGF, as the primary RFD finally began shifting eastward relative to the occluding lowlevel circulation. In contrast to the previous mesocyclone cycle, the FFCB was associated with substantial convergence throughout the development of the third mesocyclone (Figures $14(\mathrm{~h})-14(\mathrm{l}))$. The enhanced asymptotic contraction rate 


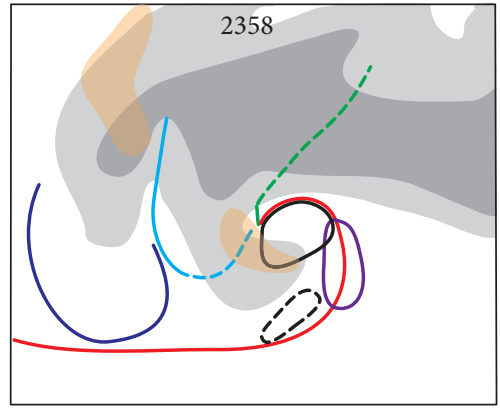

(a)

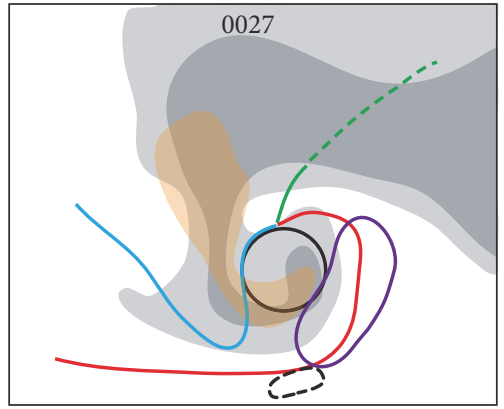

(d)

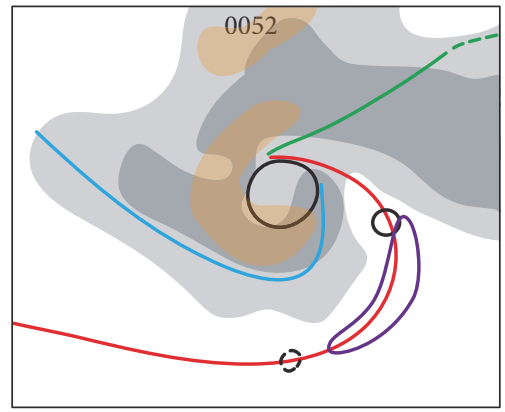

(g)

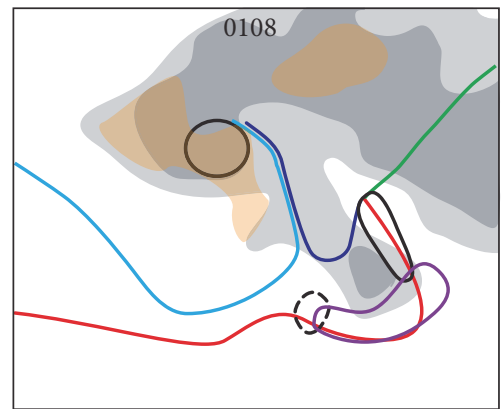

(j)

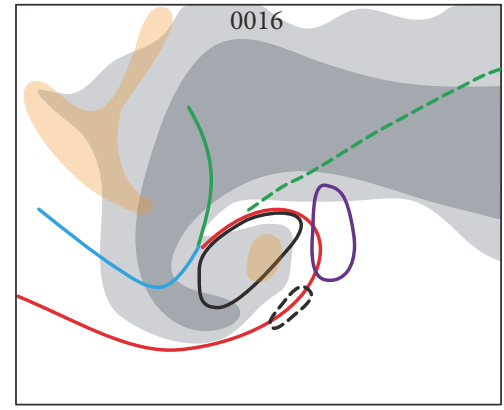

(b)

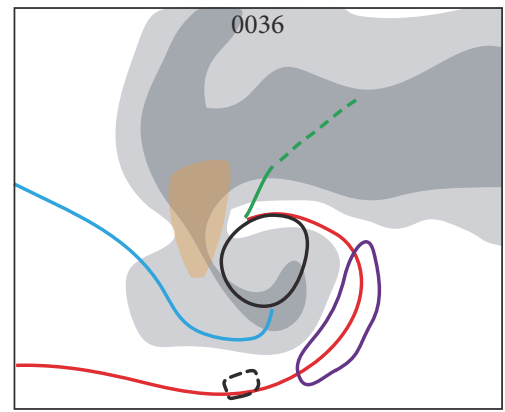

(e)

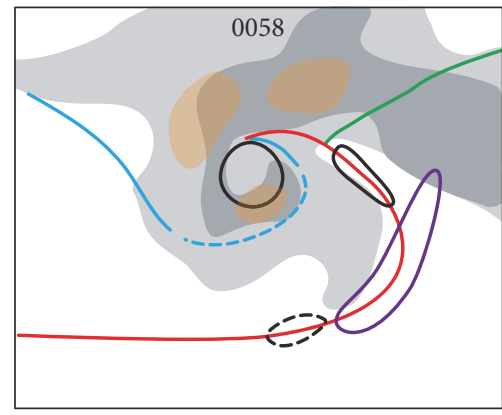

(h)

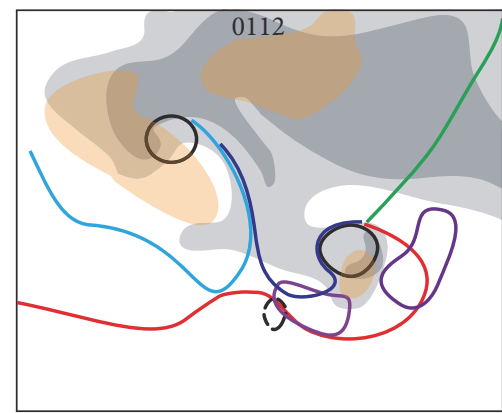

(k)

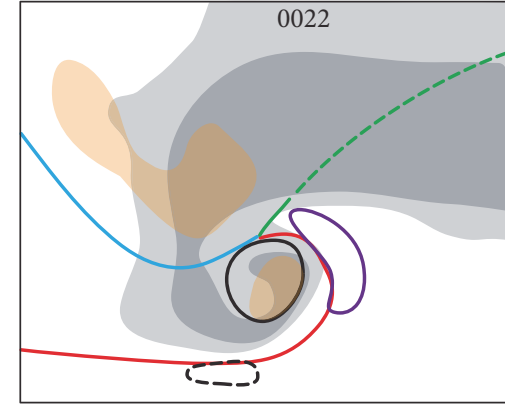

(c)

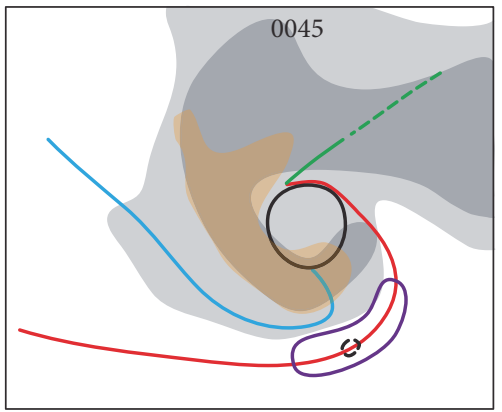

(f)

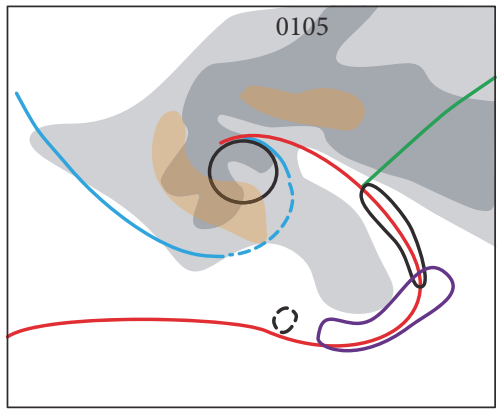

(i)

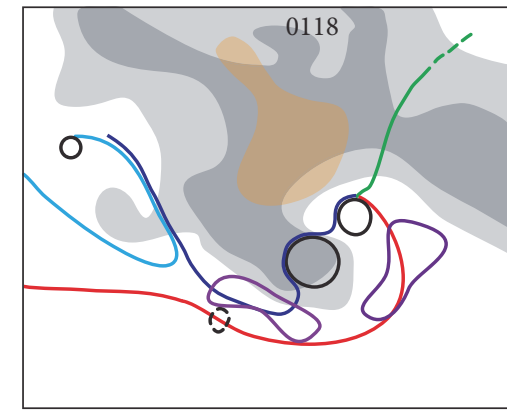

(l)

FIGURE 14: Near-surface boundaries are outlined in solid colors: red is the primary RFGF, green is the FFCB, light blue is the SRFGF, and dark blue is the new SRFGF. The near-surface mesocyclones are indicated in black ovals; the midlevel updraft shear region vorticity is indicated by purple ovals. Reflectivity was shaded grey at 40 and dark gray at $50 \mathrm{dBZ}$, and downdraft stronger than $-5 \mathrm{~m} \mathrm{~s}^{-1}$ at an altitude of $2 \mathrm{~km}$ is shaded in yellow.

and northerly low-level winds (Figure 12) suggest that the cold pool underneath the RFD was potentially stronger than during the previous cycle.

The elongated vorticity region then formed into the new mesocyclone vortex as the SRFGF surged southward, much earlier than the previous cycle, and eventually wrapped around the southern end of the new vortex (Figure 14(1)). The differences were most likely the result of the stormscale organizing stage structures propagating forward in time. However, the differing outflow evolution could also be due to 


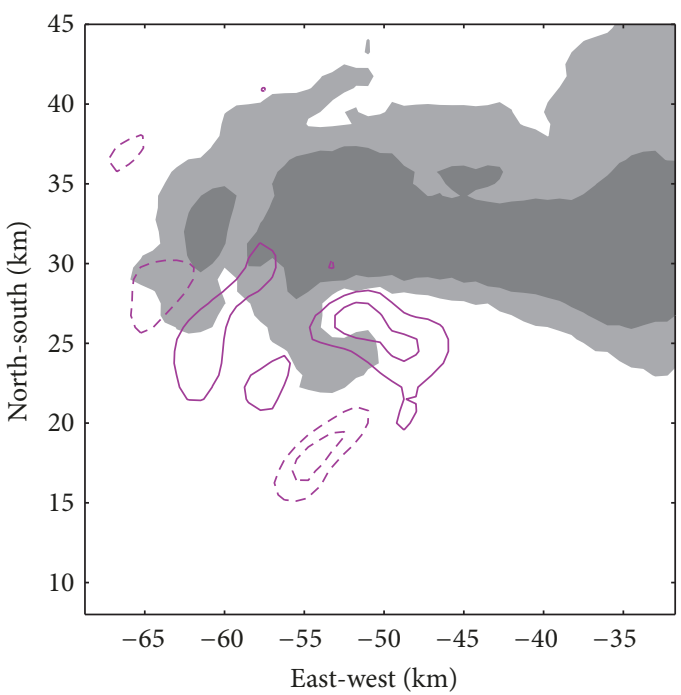

(a)

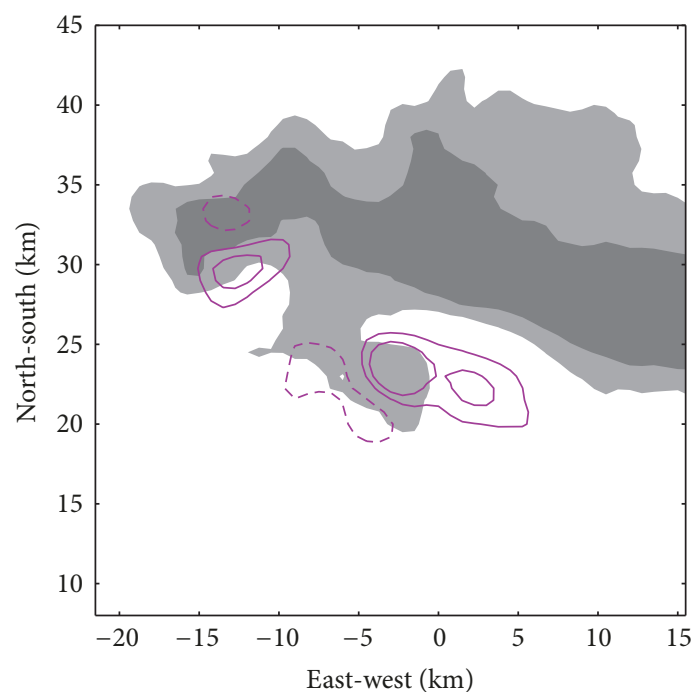

(b)

FIGURE 15: Horizontal cross-section at $250 \mathrm{~m}$ altitude of reflectivity at 30 (contoured in grey) and $50 \mathrm{dBZ}$ (contoured in dark grey) with $0-5 \mathrm{~km}$ average vertical vorticity contoured in magenta every $5 \times 10^{-3} \mathrm{~s}^{-1}$ at (a) 2358 UTC 29 May 2004 and (b) 0112 UTC on 30 May 2004 . Solid (dashed) contours denote positive (negative) values of average vertical vorticity.

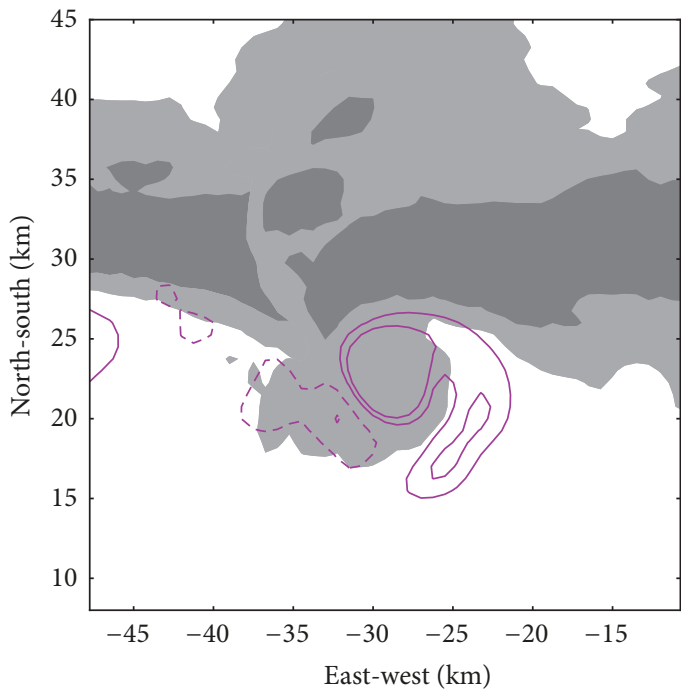

(a)

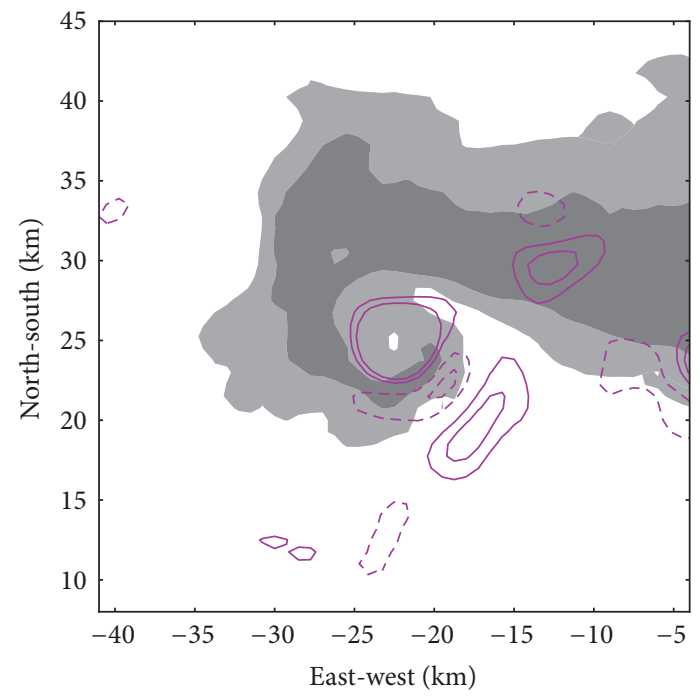

(b)

FIGURE 16: Horizontal cross-section at $250 \mathrm{~m}$ altitude of reflectivity at 30 (contoured in grey) and $50 \mathrm{dBZ}$ (contoured in dark grey) with 0-5 km average vertical vorticity contoured in magenta every $5 \times 10^{-3} \mathrm{~s}^{-1}$ at (a) 0036 UTC and (b) 0045 UTC on 30 May 2004. Solid (dashed) contours denote positive (negative) values of average vertical vorticity.

the increasing magnitudes of the capping inversion and lowlevel storm-relative helicity towards the east. Nevertheless, the new mesocyclone produced tornadoes at 0145 and 0204 UTC [60], with similar reflectivity evolution during the occlusion process (0145-0220 UTC).

\section{Conclusions}

On 29-30 May 2004, two C-Band, mobile Doppler SMART radars observed a tornadic supercell near Geary, OK, for three hours. Deep, volumetric sector scans allowed for dualDoppler analyses to be generated for an unprecedented ninety-minute period up to $18 \mathrm{~km}$ in height and over a $100 \mathrm{~km}$ by $90 \mathrm{~km}$ area horizontally. The Geary supercell was an exceptional storm in size and flash rates [50] and produced tornadoes over a seven-hour period [60], including during all three mesocyclone cycles observed here which covered the dissipating stage of the first to the organizing stage of the third cycle. The mesocyclone cycles lasted an exceptionally long (seventy-minute) period each, resulting in slower storm-scale 
evolution than most previously observed storms. Despite the cycle duration, the prolonged observational length allowed for the comparison of two organizing stages of mesocyclone development.

In general, the primary RFD was continuously found to the north or northwest of the low-level circulation during the organizing and mature stages. However, during the occlusion stage, the downdraft expanded and merged with the occlusion downdraft wrapping around the circulation. As the occlusion process progressed, the RFD shifted northeastwards as it maintained its position relative to the primary updraft and the occluding circulation moved rearward in the storm. Thus, the rear-flank and the occlusion downdrafts were generally manifested as spatially separate downdrafts for cycles two and three, until the occlusion stage, almost fifty minutes into the cycle. At middle levels, the updraft continuously surrounded the hook echo on the right and left flanks, potentially limiting evaporation due to the mixing of dry air with the environment on the western edge of the hook echo precipitation core.

Secondary RFD gust fronts (SRFGFs) were found on the western side of the circulation for all three mesocyclone cycles, including most of the second cycle that was well sampled. It is important to note that the SRFGFs did not reach the primary RFD gust front (RFGF) until the occlusion stage. During the mature and occluding stages of this storm, the SRFGF convergence forced a deep updraft on the southwestern side of the hook echo region, which resulted in the RFD outflow being ingested into the new updraft. During the dissipating stage of the first mesocyclone observed at 2358 and organizing stage of the third mesocyclone at 0108-0118 UTC, multiple SRFGFs were inferred simultaneously. However, the SRFGFs of the second and third cycle were different in that the SRFGF surged southward into the hook echo much earlier during the third cycle than during the second cycle.

The RFGF, SRFGF, and the FFCB all converged to a triple point on the north side of the circulation for most of the life cycle of the mesocyclone. As the mesocyclone matured during the second cycle, the triple point progressed eastward, due to the eastward progression of the RFD that was enhanced by an occlusion downdraft within the mesocyclone. It is important to note that the SRFGFs observed in this storm were associated with surges within the RFD and were not associated with distinctly separate downdrafts. While a forward flank gust front was not observed, the FFCB advanced eastward during the occlusion stage to the position that the forward flank gust front has been found in other studies.

In the classical mesocyclogenesis conceptual model [9, $17,65,67,68]$, the incipient low-level mesocyclone becomes positioned underneath the primary updraft during the mature stage of its lifecycle, allowing it to deepen and intensify. However, the low-level evolution of the second mesocyclone presented in this study is more similar to the alternative kinematic progression documented by Dowell and Bluestein [6]. In their conceptual model, a small portion of the updraft, here described as an occlusion updraft, separated from the primary updraft during the mature stages of the mesocyclones and provided the necessary stretching for intensification and tornadogenesis. Similarly here, the low-level mesocyclone was not connected to the primary updraft during the end of the first and all of the observed portion of the second cycle of this storm. Instead, the lowlevel mesocyclone was connected to vorticity aloft that was continuously associated with the occlusion updraft, separated in space from the primary updraft region. Thus, the second mesovortex was stretched by vertical gradients in an occlusion updraft such that maximum vorticity was nearly constant in height and the resultant erect vortex reached a maximum depth of $13 \mathrm{~km}$. The updraft shear region also contained deep, strong vorticity. However, this vorticity generally did not extend down to the surface and it remained separate from the low-level mesocyclone during the portions of the second cycle that were observed.

In contrast, the incipient and organizing stages of the third mesocyclone better followed the classical evolution. The third low-level mesocyclone developed in a vorticity-rich region underneath the vorticity associated with the updraft shear region. This mesocyclone benefited from stretching by vertical velocity gradients in the primary updraft. Hence, during the early-to-middle part of the organizing stage of the third mesocyclone, the low-level vorticity was connected to the midlevel vorticity associated with the primary updraft. However, the final analysis at 0118 UTC suggests that the new mesocyclone was in the process of becoming disconnected from the primary updraft region vorticity. Since the incipient stage of the second mesocyclone was not well sampled, it is not clear if the previous cycle transitioned from being associated with the primary updraft to being separated. Regardless, it is clear that the second mesocyclone's intensification was due to an occlusion-type updraft and not the main updraft along the primary RFGF. The analyses documented here will provide a framework for future studies that examine differences in storm-scale kinematic structure between tornadic and nontornadic supercells, particularly as more long-duration supercell data sets are collected and analyzed. Additionally, the trajectory mapping method developed by Betten et al. [69] and the diabatic Lagrangian analysis developed by Ziegler $[57,58]$ will be used to elucidate the source regions of air for the low-level rear-flank and occlusion downdrafts that other studies have shown to drive the primary and secondary gust fronts. Source regions for the mesocyclone vortex and the midlevel updraft shear region will also be investigated to understand the different sources of vorticity and tilting mechanisms.

\section{Conflicts of Interest}

The authors declare that they have no conflicts of interest.

\section{Acknowledgments}

Primary support for the radar data collection was provided by the University of Oklahoma School of Meteorology and personal funds from the corresponding author. Additional radar support was provided by a NOAA/USWRP grant to the third author, which also funded the mobile soundings. 
Data analysis was supported by the National Science Foundation under Grants nos. AGS-1063537 and AGS-1759479. The authors gratefully acknowledge the assistance of Edward (Ted) Mansell in operating the mobile sounding system.

\section{References}

[1] R. J. Trapp, "Observations of nontornadic low-level mesocyclones and attendant tornadogenesis failure during VORTEX," Monthly Weather Review, vol. 127, no. 7, pp. 1693-1710, 1999.

[2] R. M. Wakimoto and H. Cai, "Analysis of a nontornadic storm during VORTEX 95," Monthly Weather Review, vol. 128, no. 3, pp. 565-592, 2000.

[3] R. J. Trapp, G. J. Stumpf, and K. L. Manross, "A reassessment of the percentage of tornadic mesocyclones," Weather and Forecasting, vol. 20, no. 4, pp. 680-687, 2005.

[4] D. L. Darkow and C. J. Roos, "Multiple tornado producing thunderstorms and their apparent cyclic variations in intensity," in Proceedings of the Preprints Fourteenth Conf. on Radar Meteorology, pp. 305-308, 1970.

[5] D. W. Burgess, V. T. Wood, and R. A. Brown, "Mesocyclone evolution statistics," in Preprints, 10th Conf. on Severe Local Storms, pp. 422-424, Amer. Meteor. Soc., Omaha, NE, USA, 1982.

[6] D. C. Dowell and H. B. Bluestein, "The 8 June 1995 McLean, Texas, storm. Part I: Observations of cyclic tornadogenesis," Monthly Weather Review, vol. 130, no. 11, pp. 2626-2648, 2002.

[7] J. R. Beck, J. L. Schroeder, and J. M. Wurman, "High-resolution dual-Doppler analyses of the 29 May 2001 Kress, Texas, cyclic supercell," Monthly Weather Review, vol. 134, no. 11, pp. 3125$3148,2006$.

[8] M. M. French, H. B. Bluestein, D. C. Dowell, L. J. Wicker, M. R. Kramar, and A. L. Pazmany, "High-resolution, mobile Doppler radar observations of cyclic mesocyclogenesis in a supercell," Monthly Weather Review, vol. 136, no. 12, pp. 4997-5016, 2008.

[9] E. J. Adlerman, K. K. Droegemeier, and R. Davies-Jones, "A numerical simulation of cyclic mesocyclogenesis," Journal of the Atmospheric Sciences, vol. 56, no. 13, pp. 2045-2069, 1999.

[10] E. J. Adlerman and K. K. Droegemeier, "The sensitivity of numerically simulated cyclic mesocyclogenesis to variations in model physical and computational parameters," Monthly Weather Review, vol. 130, no. 11, pp. 2671-2691, 2002.

[11] E. J. Adlerman and K. K. Droegemeier, "The dependence of numerically simulated cyclic mesocyclogenesis upon environmental vertical wind shear," Monthly Weather Review, vol. 133, no. 12, pp. 3595-3623, 2005.

[12] T. Fujita, "Proposed mechanism of tornado formation from rotating thunderstorm," in Preprints, 8th Conf. on Severe Local Storms, pp. 191-196, Amer. Meteor. Soc., Denver, CO, USA, 1973.

[13] E. M. Agee, J. T. Snow, and P. R. Clare, "Multiple vortex features in the tornado cyclone and the occurrence of tornado families," Monthly Weather Review, vol. 104, no. 5, pp. 552-563, 1976.

[14] B. Jensen, T. P. Marshall, M. A. Mabey, and E. N. Rasmussen, "Storm scale structure of the Pampa storm," in Preprints, 13th Conf. on Severe Local Storms, pp. 85-88, Tulsa, OK, USA, 1983.

[15] K. W. Johnson, P. S. Ray, B. C. Johnson, and R. P. Davies-Jones, "Observations related to the rotational dynamics of the 20 May 1977 tornadic storms," Mon. Wea. Rev, vol. 115, pp. 2463-2478, 1987.

[16] K. R. Knupp, T. A. Murphy, T. A. Coleman et al., "Meteorological overview of the devastating 27 April 2011 tornado outbreak,"
Bulletin of the American Meteorological Society, vol. 95, no. 7, pp. 1041-1062, 2014.

[17] L. R. Lemon and C. A. Doswell III, "Severe thunderstorm evolution and mesocyclone structure as related to tornadogenesis.", Monthly Weather Review, vol. 107, no. 9, pp. 1184-1197, 1979.

[18] D. C. Dowell and H. B. Bluestein, “The 8 June 1995 McLean, Texas, storm. Part II: Cyclic tornado formation, maintenance, and dissipation," Monthly Weather Review, vol. 130, no. 11, pp. 2649-2670, 2002.

[19] C. J. Shabbott and P. M. Markowski, "Surface in situ observations within the outflow of forward-flank downdrafts of supercell thunderstorms," Monthly Weather Review, vol. 134, no. 5, pp. 1422-1441, 2006.

[20] P. S. Skinner, C. C. Weiss, J. L. Schroeder, L. J. Wicker, and M. I. Biggerstaff, "Observations of the surface boundary structure within the 23 May 2007 Perryton, Texas, supercell," Monthly Weather Review, vol. 139, no. 12, pp. 3730-3749, 2011.

[21] P. Markowski, Y. Richardson, J. Marquis et al., "The pretornadic phase of the Goshen County, Wyoming, supercell of 5 June 2009 intercepted by VORTEX2. Part I: Evolution of kinematic and surface thermodynamic fields," Monthly Weather Review, vol. 140, no. 9, pp. 2887-2915, 2012.

[22] C. C. Weiss, D. C. Dowell, J. L. Schroeder et al., "A comparison of near-surface buoyancy and baroclinity across three VORTEX2 supercell intercepts," Monthly Weather Review, vol. 143, no. 7, pp. 2736-2753, 2015.

[23] J. B. Klemp and R. Rotunno, "A study of the tornadic region within a supercell thunderstorm.," Journal of the Atmospheric Sciences, vol. 40, no. 2, pp. 359-377, 1983.

[24] E. A. Brandes, "Mesocyclone evolution and tornadogenesis: some observations," Monthly Weather Review, vol. 106, no. 7, pp. 995-1011, 1978.

[25] D. C. Dowell and H. B. Bluestein, "The Arcadia, Oklahoma, storm of 17 May 1981: Analysis of a supercell during tornadogenesis," Monthly Weather Review, vol. 125, no. 10, pp. 2562-2582, 1997.

[26] R. M. Wakimoto and C. Liu, "The Garden City, Kansas, storm during VORTEX 95. Part II: The wall cloud and tornado," Monthly Weather Review, vol. 126, no. 2, pp. 393-408, 1998.

[27] C. L. Ziegler, E. N. Rasmussen, T. R. Shepherd, A. I. Watson, and J. M. Straka, "The evolution of low-level rotation in the 29 May 1994 Newcastle-Graham, Texas, storm complex during VORTEX," Monthly Weather Review, vol. 129, no. 6, pp. 13391368, 2001.

[28] J. Marquis, Y. Richardson, P. Markowski, D. Dowell, and J. Wurman, "Tornado maintenance investigated with high-resolution dual-Doppler and EnKF analysis," Monthly Weather Review, vol. 140, no. 1, pp. 3-27, 2012.

[29] K. Kosiba, J. Wurman, Y. Richardson, P. Markowski, P. Robinson, and J. Marquis, "Genesis of the Goshen County, Wyoming, tornado on 5 June 2009 during VORTEX2," Monthly Weather Review, vol. 141, no. 4, pp. 1157-1181, 2013.

[30] P. M. Markowski, "Mobile mesonet observations on 3 May 1999," Weather and Forecasting, vol. 17, no. 3, pp. 430-444, 2002.

[31] P. M. Markowski, J. M. Straka, and E. N. Rasmussen, "Direct surface thermodynamic observations within the rear-flank downdrafts of nontornadic and tornadic supercells," Monthly Weather Review, vol. 130, no. 7, pp. 1692-1721, 2002.

[32] B. D. Lee, C. A. Finley, and C. D. Karstens, "The Bowdle, South Dakota, cyclic tornadic supercell of 22 May 2010: Surface analysis of rear-flank downdraft evolution and multiple internal 
surges," Monthly Weather Review, vol. 140, no. 11, pp. 3419-3441, 2012.

[33] C. A. Finley and B. D. Lee, "High resolution mobile mesonet observations of RFD surges in the June 9 Basset, Nebraska supercell during project ANSWERS," in Extended Abstracts, 22nd Conf. on Severe Local Storms, Hyannis, MA, Amer. Meteor. Soc, 2004, http://ams.confex.com/ams/1laram22sls/techprogram/ paper_82005.htm.

[34] C. A. Finley and B. D. Lee, "Mobile mesonet observations of an intense RFD and multiple RFD gust fronts in the May 23 Quinter, Kansas tornadic supercell during TWISTEX," in Proceedings of the Preprints, 24th Conf. on Severe Local Storms, Savannah, GA, Amer. Meteor. Soc, 2008, http://ams.confex.com/ams/ pdfpapers/142233.pdf.

[35] B. D. Hirth, J. L. Schroeder, and C. C. Weiss, "Surface analysis of the rear-flank downdraft outflow in two tornadic supercells," Monthly Weather Review, vol. 136, no. 7, pp. 2344-2363, 2008.

[36] B. D. Lee, C. A. Finley, and T. M. Samaras, "Surface analysis near and within the Tipton, Kansas, tornado on 29 May 2008," Monthly Weather Review, vol. 139, no. 2, pp. 370-386, 2011.

[37] J. Wurman, Y. Richardson, C. Alexander, S. Weygandt, and P. F. Zhang, "Dual-Doppler and single-Doppler analysis of a tornadic storm undergoing mergers and repeated tornadogenesis," Monthly Weather Review, vol. 135, no. 3, pp. 736-758, 2007.

[38] J. Marquis, Y. Richardson, J. Wurman, and P. Markowski, "Single- and dual-Doppler analysis of a tornadic vortex and surrounding storm-scale flow in the Crowell, Texas, supercell of 30 April 2000," Monthly Weather Review, vol. 136, no. 12, pp. 5017-5043, 2008.

[39] J. Wurman, K. Kosiba, P. Markowski, Y. Richardson, D. Dowell, and P. Robinson, "Finescale single- and dual-Doppler analysis of tornado intensification, maintenance, and dissipation in the Orleans, Nebraska, supercell," Monthly Weather Review, vol. 138, no. 12, pp. 4439-4455, 2010.

[40] C. J. Riganti and A. L. Houston, "Rear-flank outflow dynamics and thermodynamics in the 10 June 2010 Last Chance, Colorado, supercell," Monthly Weather Review, vol. 145, no. 7, pp. 2487-2504, 2017

[41] P. S. Skinner, C. C. Weiss, M. M. French, H. B. Bluestein, P. M. Markowski, and Y. P. Richardson, "VORTEX2 observations of a low-level mesocyclone with multiple internal rear-flank downdraft momentum surges in the 18 May 2010 Dumas, Texas, supercell," Monthly Weather Review, vol. 142, no. 8, pp. 29352960, 2014.

[42] P. S. Skinner, C. C. Weiss, L. J. Wicker, C. K. Potvin, and D. C. Dowell, "Forcing mechanisms for an internal rear-flank downdraft momentum surge in the 18 May 2010 Dumas, Texas, supercell," Monthly Weather Review, vol. 143, no. 11, pp. 43054330, 2015.

[43] W. Mashiko, H. Niino, and T. Kato, "Numerical simulation of tornadogenesis in an outer-rainband minisupercell of Typhoon Shanshan on 17 September 2006," Monthly Weather Review, vol. 137, no. 12, pp. 4238-4260, 2009.

[44] A. D. Schenkman, M. Xue, and D. T. Dawson, "The cause of internal outflow surges in a high-resolution simulation of the 8 May 2003 Oklahoma City tornadic supercell," Journal of the Atmospheric Sciences, vol. 73, no. 1, pp. 353-370, 2016.

[45] D. R. MacGorman, W. David Rust, T. J. Schuur et al., “TELEX The Thunderstorm Electrification and Lightning Experiment," Bulletin of the American Meteorological Society, vol. 89, no. 7, pp. 997-1013, 2008.
[46] M. I. Biggerstaff, L. J. Wicker, J. Guynes et al., "The shared mobile atmospheric research and teaching radar: A collaboration to enhance research and teaching," Bulletin of the American Meteorological Society, vol. 86, no. 9, pp. 1263-1274, 2005.

[47] E. A. Brandes, "Gust front evolution and tornado genesis as viewed by Doppler radar," Journal of Applied Meteorology and Climatology, vol. 16, no. 4, pp. 333-338, 1977.

[48] P. S. Ray, "The morphology of several tornadic storms on 20 May 1977.", Journal of the Atmospheric Sciences, vol. 38, no. 8, pp. 1643-1663, 1981.

[49] M. I. Biggerstaff and R. A. Houze Jr., "Kinematics and microphysics of the transition zone of the 10-11 June 1985 squall line," Journal of the Atmospheric Sciences, vol. 50, no. 18, pp. 3091-3110, 1993.

[50] K. M. Calhoun, D. R. MacGorman, C. L. Ziegler, and M. I. Biggerstaff, "Evolution of lightning activity and storm charge relative to dual-Doppler analysis of a high-precipitation supercell storm," Monthly Weather Review, vol. 141, no. 7, pp. 21992223, 2013.

[51] G. P. Cressman, "An operational objective analysis system," Monthly Weather Review, vol. 87, no. 10, pp. 367-374, 1959.

[52] A. D. Gordon and V. Barnett, "Interpreting multivariate data," Biometrics, vol. 38, no. 3, p. 869, 1982.

[53] C. G. Mohr, L. Jay Miller, R. L. Vaughan, and H. W. Frank, “The merger of mesoscale datasets into a common Cartesian format for efficient and systematic analyses," Journal of Atmospheric and Oceanic Technology, vol. 3, no. 1, pp. 143-161, 1986.

[54] J. L. Palucki, M. I. Biggerstaff, D. R. MacGorman, and T. Schuur, "Comparison between low-flash and non-lightning-producing convective areas within a mature mesoscale convective system," Weather and Forecasting, vol. 26, no. 4, pp. 468-486, 2011.

[55] J. A. Leise, "A multidimensional scale-telescoped filter and data extension package," in NOAA Tech. Memo. ERL WPL-82, pp. 18 82, 1981.

[56] R. A. Cohen and D. M. Schultz, "Contraction rate and its relationship to frontogenesis, the Lyapunov exponent, fluid trapping, and airstream boundaries," Monthly Weather Review, vol. 133, no. 5, pp. 1353-1369, 2005.

[57] C. L. Ziegler, "A diabatic Lagrangian technique for the analysis of convective storms. Part I: Description and validation via an observing system simulation experiment," Journal of Atmospheric and Oceanic Technology, vol. 30, no. 10, pp. 2248-2265, 2013.

[58] C. L. Ziegler, "A diabatic Lagrangian technique for the analysis of convective storms. Part II: Application to a radar-observed storm," Journal of Atmospheric and Oceanic Technology, vol. 30, no. 10, pp. 2266-2280, 2013.

[59] A. R. Moller, C. A. Doswell, M. P. Foster, and G. R. Woodall, "The operational recognition of supercell thunderstorm environments and storm structures," Weather and Forecasting, vol. 9, no. 3, pp. 327-347, 1994.

[60] NCDC, 2004: Storm Data. Vol. 48, No. 6, pp. 486.

[61] J. Wurman, J. Straka, E. Rasmussen, M. Randall, and A. Zahrai, "Design and deployment of a portable, pencil-beam, pulsed, 3-cm Doppler radar," Journal of Atmospheric and Oceanic Technology, vol. 14, no. 6, pp. 1502-1512, 1997.

[62] C. L. Ziegler, E. R. Mansell, J. M. Straka, D. R. MacGorman, and D. W. Burgess, "The impact of spatial variations of lowlevel stability on the life cycle of a simulated supercell storm," Monthly Weather Review, vol. 138, no. 5, pp. 1738-1766, 2010. 
[63] R. L. Thompson, R. Edwards, J. A. Hart, K. L. Elmore, and P. Markowski, "Close proximity soundings within supercell environments obtained from the rapid update cycle," Weather and Forecasting, vol. 18, no. 6, pp. 1243-1261, 2003.

[64] J. Beck and C. Weiss, "An assessment of low-level baroclinity and vorticity within a simulated supercell," Monthly Weather Review, vol. 141, no. 2, pp. 649-669, 2013.

[65] P. M. Markowski and Y. P. Richardson, "The influence of environmental low-level shear and cold pools on tornadogenesis: Insights from idealized simulations," Journal of the Atmospheric Sciences, vol. 71, no. 1, pp. 243-275, 2014.

[66] J. B. Klemp, R. B. Wilhelmson, and P. S. Ray, "Observed and numerically simulated structure of a mature supercell thunderstorm (Del City, Oklahoma)," Journal of the Atmospheric Sciences, vol. 38, no. 8, pp. 1558-1580, 1981.

[67] P. Markowski, E. Rasmussen, J. Straka, R. Davies-Jones, Y. Richardson, and R. J. Trapp, "Vortex lines within lowlevel mesocyclones obtained from pseudo-dual-Doppler radar observations," Monthly Weather Review, vol. 136, no. 9, pp. 35133535, 2008.

[68] R. Rotunno and J. B. Klemp, "On the rotation and propagation of simulated supercell thunderstorms.", Journal of the Atmospheric Sciences, vol. 42, no. 3, pp. 271-292, 1985.

[69] D. P. Betten, M. I. Biggerstaff, and L. J. Wicker, "A trajectory mapping technique for the visualization and analysis of threedimensional flow in supercell storms," Journal of Atmospheric and Oceanic Technology, vol. 34, no. 1, pp. 33-49, 2017. 

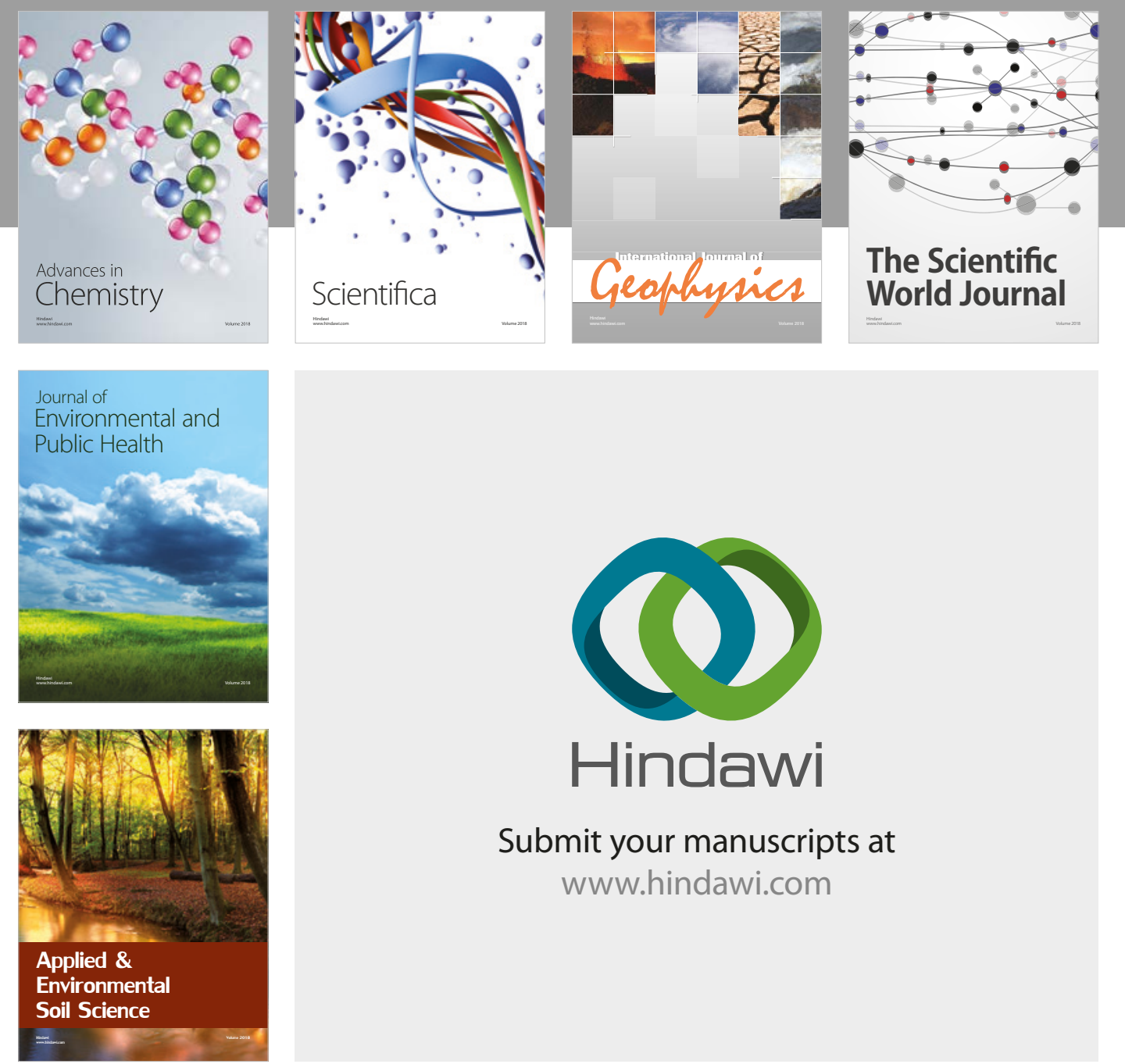

The Scientific

\section{World Journal}
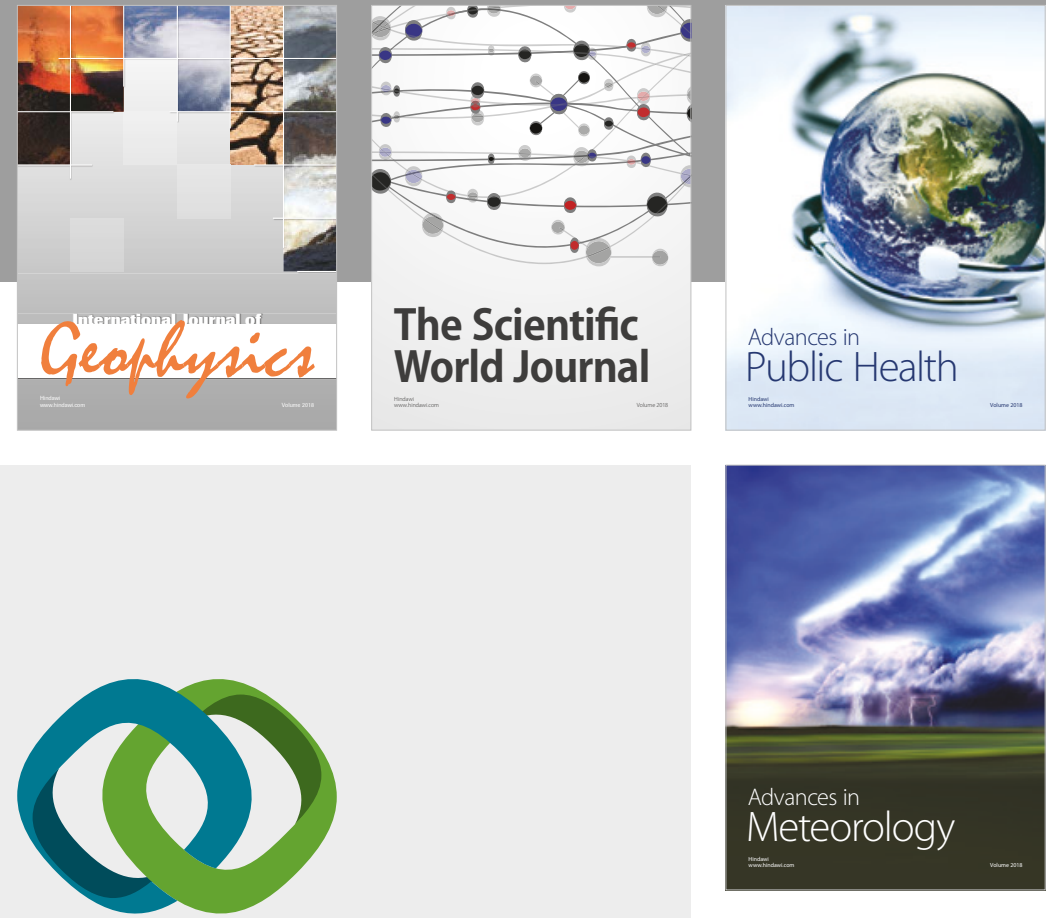

Advan

Public Health

\section{Hindawi}

Submit your manuscripts at

www.hindawi.com
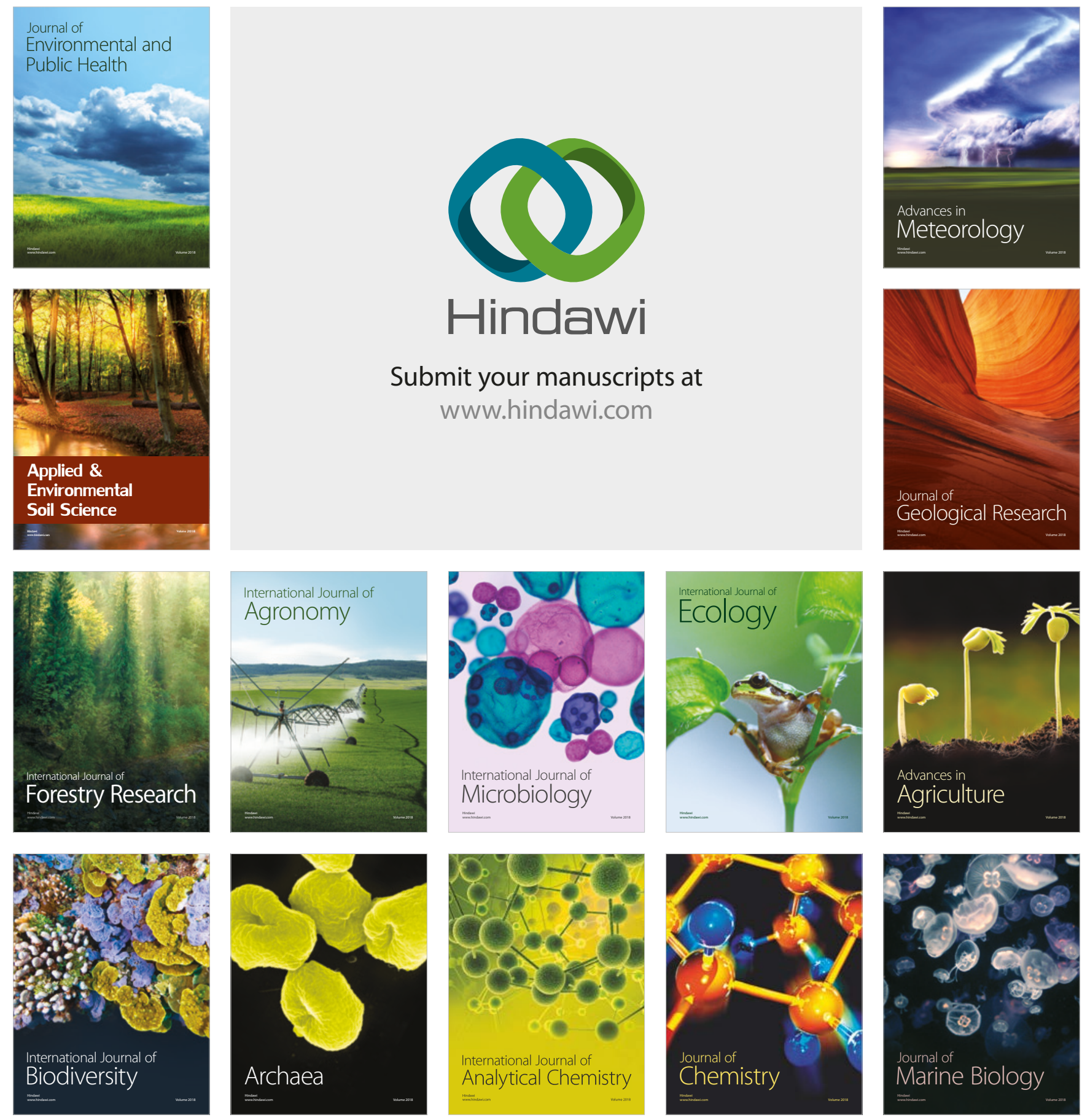